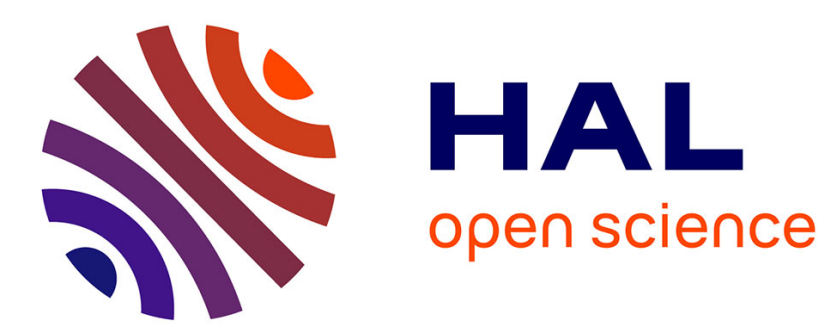

\title{
New insights on marginally trapped surfaces: the hedgehog theory point of view
}

\author{
Yves Martinez-Maure
}

\section{To cite this version:}

Yves Martinez-Maure. New insights on marginally trapped surfaces: the hedgehog theory point of view. 2018. hal-01627452v2

\section{HAL Id: hal-01627452 \\ https://hal.science/hal-01627452v2}

Preprint submitted on 31 Aug 2018

HAL is a multi-disciplinary open access archive for the deposit and dissemination of scientific research documents, whether they are published or not. The documents may come from teaching and research institutions in France or abroad, or from public or private research centers.
L'archive ouverte pluridisciplinaire HAL, est destinée au dépôt et à la diffusion de documents scientifiques de niveau recherche, publiés ou non, émanant des établissements d'enseignement et de recherche français ou étrangers, des laboratoires publics ou privés. 


\title{
New insights on marginally trapped surfaces: the hedgehog theory point of view
}

\author{
Yves Martinez-Maure \\ yves.martinez-maure@imj-prg.fr
}

October 21, 2017

\begin{abstract}
In this paper, we will try to argue and to show through fundamental examples that (a very huge class of) marginally trapped surfaces arise naturally from a 'lightlike co-contact structure', exactly in the same way as Legendrian fronts arise from a contact one (by projection of a Legendrian submanifold to the base of a Legendrian fibration), and that there is an adjunction relationship between both notions. We especially focus our interest on marginally trapped hedgehogs and study their relationships with Laguerre geometry and Brunn-Minkowski theory.
\end{abstract}

\section{Contents:}

1. Introduction and statement of main results

1.1. Marginally trapped hedgehogs or co-hedgehogs in $\mathbb{L}^{4}$

1.1.1. Characterization and definitions in $\mathbb{L}^{4}$

1.1.2. Geometrical interpretation of co-hedgehogs of $\mathbb{L}^{4}$

1.1.3. Relationship with Laguerre geometry

1.2. Relationship with the Brunn-Minkowski theory

1.3. Synthetic and comparative co-presentation of hedgehogs and (co)hedgehogs

2. Marginally trapped hedgehogs in other spaces and generalizations

2.1. Marginally trapped hedgehogs or co-hedgehogs in $\mathbf{M}^{4}$

2.1.1. Characterization and definitions in $\mathbf{M}^{4}$

2.1.2. Geometrical interpretation of co-hedgehogs of $\mathbf{M}^{4}$

2.2. Towards other spaces and generalizations

2.2.1. Basic definitions and facts

- Contact manifolds and metric contact manifolds

2010 MSC: 52A30; 52A39; 53D10; 53A35; 53A40; 53B30;

Key words and phrases: Hedgehogs, marginally trapped surfaces, Laguerre geometry, Brunn-

Minkowki theory, contact and co-contact conditions 
- Hedgehogs as Legendrian fronts

- Symplectic and almost-hermitian structures

2.2.2. Marginally trapped hedgehog of an associated Lagrangian submanifold

3. Proof of the main results

4. Further remarks and problems

4.1. Curvature function of marginally trapped hedgehogs

4.2. Minkowski problem for marginally trapped hedgehogs

\section{Introduction and statement of main results}

Trapped surfaces were introduced in general relativity by R. Penrose [12] to study singularities of spacetimes. They appeared in a natural way earlier in the work of Blaschke, in the context of conformal and Laguerre geometry [1]. These surfaces play an extremely important role in general relativity where they are of central importance in the study of black holes, those regions of spacetime where everything is trapped, and nothing can escape, even light. A closed embedded spacelike 2-surface of a 4-dimensional spacetime is said to be trapped if its mean curvature vector is everywhere timelike. The limiting case of marginally trapped surfaces, i.e. surfaces whose mean curvature vector is everywhere lightlike, play the role of apparent horizons of black holes. Mathematically, marginally trapped surfaces are regarded as spacetime analogues of minimal surfaces in Riemannian geometry. Even though they received considerable attention both from mathematicians and physicists, these surfaces are still not very well understood. For a recent survey on marginally trapped surfaces, see the book by B.Y. Chen [3].

What we will try to argue in this paper, and to show through fundamental examples, is that:

(A very huge class of) marginally trapped surfaces arise naturally from a 'lightlike co-contact structure', exactly in the same way as Legendrian fronts arise from a contact one (by projection of a Legendrian submanifold to the base of a Legendrian fibration), and there is an adjunction relationship between both notions.

In addition, a huge class of marginally trapped surfaces correspond by adjunction to hedgehogs (envelopes parametrized by their Gauss map) and can thus benefit directly from contributions of hedgehog theory, which can be seen as an extension of the Brunn-Minkowski one (see e.g. [10]). This correspondence is naturally promising in terms of new geometric inequalities, and we know how important geometric inequalities are in gravitation. We will give examples of geometric inequalities involving hedgehogs and marginally trapped surfaces in Subsection 1.2.

In order to explain precisely what we mean here by a 'lightlike co-contact structure', let us begin by the presentation of a fundamental example in the 4-dimensional Lorentz-Minkowski space $\mathbb{L}^{4}$. This example will be detailed in Subsection 1.3. 


\subsection{Marginally trapped hedgehogs or co-hedgehogs in $\mathbb{L}^{4}$}

\subsubsection{Characterization and definitions in $\mathbb{L}^{4}$}

For simplicity, we will restrict our presentation to surfaces in $\mathbb{L}^{4}$ but our results extend, without much change, to higher dimensions. To any $h \in C^{\infty}\left(\mathbb{S}^{2} ; \mathbb{R}\right)$ corresponds the envelope $\mathcal{H}_{h}$ of the family $\left(P_{h}(u)\right)_{u \in \mathbb{S}^{2}}$ of cooriented planes of $\mathbb{R}^{3}$ with equation $(E) \quad\langle x, u\rangle=h(u)$, where $\langle.,$.$\rangle is the standard scalar product$ on $\mathbb{R}^{3}$. We say that $\mathcal{H}_{h}$ is the hedgehog with support function $h$. From $(E)$ and the contact condition $\langle d x, u\rangle=0$, we deduce

$$
\left\{\begin{array}{l}
\langle x, u\rangle=h(u) \\
\langle x, d u\rangle=d h_{u},
\end{array}\right.
$$

for all $u \in \mathbb{S}^{2}$. Thus, it appears that $\mathcal{H}_{h}$ can be parametrized by $x_{h}: \mathbb{S}^{2} \rightarrow \mathbb{R}^{3}$, $u \mapsto h(u) u+(\nabla h)(u)$, where $(\nabla h)(u)$ stands for the gradient of $h$ at $u$. The parametrization $x_{h}$ can be interpreted as the inverse of its Gauss map (if $u$ is a regular point of $x_{h}$, then $u$ is normal to $\mathcal{H}_{h}$ at $\left.x_{h}(u)\right)$. Note that $\left(\mathcal{H}_{h+t}\right)_{t \in \mathbb{R}}$ is a family of parallel hedgehogs in $\mathbb{R}^{3}$ : for all $(u, t) \in \mathbb{S}^{2} \times \mathbb{R}, x_{h+t}(u)=x_{h}(u)+t u$.

Now, we claim that:

To the differential dh of $h$ corresponds naturally a 'marginally trapped hedgehog' of the Lorentz Minkowski 4-space $\mathbb{L}^{4}=\left(\mathbb{R}^{4},\langle., .\rangle_{L}\right)$ via a 'lightlike co-contact condition'.

Here, the pseudo-scalar product $\langle., .\rangle_{L}$ is defined by

$$
\left\langle(x, t),\left(x^{\prime}, t^{\prime}\right)\right\rangle_{L}:=x_{1} x_{1}^{\prime}+x_{2} x_{2}^{\prime}+x_{3} x_{3}^{\prime}-t t^{\prime},
$$

for all $(x, t):=\left(\left(x_{1}, x_{2}, x_{3}\right), t\right)$ and $\left(x^{\prime}, t^{\prime}\right):=\left(\left(x_{1}^{\prime}, x_{2}^{\prime}, x_{3}^{\prime}\right), t^{\prime}\right)$ in $\mathbb{R}^{4}=\mathbb{R}^{3} \times \mathbb{R}$. Indeed, $d h$ determines the family $\left(L_{h+t}(u)\right)_{(u, t) \in \mathbb{S}^{2} \times \mathbb{R}}$ of oriented null lines of $\mathbb{L}^{4}$ defined by:

$$
\forall(u, t) \in \mathbb{S}^{2} \times \mathbb{R}, L_{h+t}(u)=\left\{l_{h+t}(u)\right\}+\mathbb{R}(u,-1),
$$

where $l_{h+t}(u):=(\nabla h(u), h(u)+t)$; and this family of null lines determines a 'marginally trapped hedgehog', which is unique up to translations parallel to the time axis, via a 'lightlike co-contact condition':

Theorem 1 (Determination of marginally trapped hedgehogs of $\mathbb{L}^{4}$ by 1-jet and co-contact condition) For all $t \in \mathbb{R},(i)$ there is a unique map $x$ : $\mathbb{S}^{2} \rightarrow \mathbb{L}^{4}$ of class $C^{\infty}$ satisfying $x(u) \in L_{h+t}(u)$ for all $u \in \mathbb{S}^{2}$ together with the 'lightlike co-contact condition'

$$
\left\langle\delta(d x), u_{L}\right\rangle_{L}=0,
$$

where $\delta$ is the Hodge codifferential on $\mathbb{S}^{2}$ (i.e. the formal adjoint to the exterior differentiation $d$ ) and $u_{L}:=\frac{1}{\sqrt{2}}(u,-1)$, namely

$$
x=x_{l_{h+t}}: \mathbb{S}^{2} \rightarrow \mathbb{L}^{4}, u \mapsto l_{h+t}(u)+\delta(\partial h)(u) u_{L},
$$


where $\partial h(u):=d h / \sqrt{2}$;

(ii) this map $x: \mathbb{S}^{2} \rightarrow \mathbb{L}^{4}$ is such that $x^{*} g=\frac{1}{4}\left(R_{1}-R_{2}\right)^{2} g_{S}$, where $g_{S}$ is the standard metric on $\mathbb{S}^{2}, g$ the first fundamental form on $x\left(\mathbb{S}^{2}\right), x^{*} g$ the pullback of $g$ along $x$ and $R_{1}(u), R_{2}(u)$ the principal radii of curvature of $\mathcal{H}_{h}$ at $x_{h}(u)$;

(iii) for all $u \in \mathbb{S}^{2}, \Delta x(u) \in \mathbb{R} u_{L}$, where $\Delta$ is the Hodge Laplacian on $\mathbb{S}^{2}$, so that the mean curvature vector of $x\left(\mathbb{S}^{2}\right)$ at $x(u)$, say $\vec{H}_{x}(u)$, is parallel to the lightlike vector $\overrightarrow{u_{L}}$ whenever $x_{h}(u)$ is not an umbilical point of $\mathcal{H}_{h}$, that is:

$$
\forall u \in \mathbb{S}^{2} \backslash\left\{u \in \mathbb{S}^{2} \mid R_{1}(u)=R_{2}(u)\right\}, \quad \vec{H}_{x}(u) \in \mathbb{R} \overrightarrow{u_{L}}
$$

Of course, the Hodge-Laplacian of a $C^{\infty}$-map $x: \mathbb{S}^{2} \rightarrow \mathbb{L}^{4}, u \mapsto\left(x_{i}(u)\right)_{i=1}^{4}$ is understood to be the vector function $\Delta X: \mathbb{S}^{2} \rightarrow \mathbb{L}^{4}, u \mapsto\left(\Delta x_{i}(u)\right)_{i=1}^{4}$, where $\Delta x_{i}$ is the Hodge-Laplacian of the coordinate function $x_{i}$ in the intrinsic metric on $\mathbb{S}^{2},(1 \leq i \leq 4)$.

Definition 1. For all $t \in \mathbb{R}$, we call $\mathcal{H}_{l_{h+t}}:=x_{l_{h+t}}\left(\mathbb{S}^{2}\right)$ the (marginally trapped) hedgehog with support 1 -jet $l_{h+t}: \mathbb{S}^{2} \rightarrow J^{1}\left(\mathbb{S}^{2}\right), u \mapsto(\nabla h(u), h(u)+t)$.

Note that $\sqrt{2} \partial$ stands for the Hodge-Dirac operator $D=d+\delta$ on $\mathbb{S}^{2}$ and that the datum of $\partial h:=d h / \sqrt{2}$ is equivalent to the one of the family of 1 -jets $\left(l_{h+t}\right)_{t \in \mathbb{R}}$, where $l_{h+t}:=(\nabla h, h+t)$. All the marginally trapped hedgehogs of the family $\left(\mathcal{H}_{l_{h+t}}\right)_{t \in \mathbb{R}}$ are equal up to translations parallel to the time axis $\mathbb{R} \overrightarrow{\partial_{t}}$ in $\mathbb{L}^{4}$, where $\overrightarrow{\partial_{t}}:=\left(\overrightarrow{\mathbb{R}^{3}}, 1\right)$, since: for all $(u, t) \in \mathbb{S}^{2} \times \mathbb{R}, x_{l_{h+t}}(u)=x_{l_{h}}(u)+t \overrightarrow{\partial_{t}}$. Therefore, this family can be regarded as one and only one marginally trapped hedgehog defined up to a translation parallel to the time axis $\mathbb{R} \overrightarrow{\partial_{t}}$ in $\mathbb{L}^{4}$. This hedgehog will be denoted by $\mathcal{H}_{\partial h}$, where $\partial h:=d h / \sqrt{2}$. The factor $1 / \sqrt{2}$ is chosen so that the hedgehog with support function $\partial^{2} h:=\partial(\partial h)$ coincides with the mean evolute of $\mathcal{H}_{h}$ (see Subsection 1.2 for details and a precise definition of the mean evolute).

These remarks make natural the following definition:

Definition 2. We call $\mathcal{H}_{\partial h}$ the marginally trapped hedgehog (or, co-hedgehog) of $\mathbb{L}^{4}$ with support differential $\partial h:=d h / \sqrt{2}$. Anyone of the marginally trapped hedgehogs $\mathcal{H}_{l_{h+t}},(t \in \mathbb{R})$, will be regarded as a representative of $\mathcal{H}_{\partial h}$ in $\mathbb{L}^{4}$. We will say that $\mathcal{H}_{\partial h}$ is the co-evolute of $\mathcal{H}_{h}$ in $\mathbb{L}^{4}$.

Thus, once the lightlike 'co-contact condition' $\left\langle\delta(d x), u_{L}\right\rangle_{L}=0$ is fixed, the (co)hedgehog $\mathcal{H}_{\partial h}$ is defined in $\mathbb{L}^{4}$ as the 'co-envelope' of the family of oriented null lines determined by its support differential $\partial h$ in the same way as, once the contact condition $\langle d x, u\rangle=0$ is fixed, the hedgehog $\mathcal{H}_{h}$ is defined in $\mathbb{R}^{3}$ as the envelope of the family of cooriented planes determined by its support function $h$. Of course, it is important to note that $\mathcal{H}_{h}$ and $\mathcal{H}_{\partial h}$ are not assumed 
to be embedded surfaces of respectively $\mathbb{R}^{3}$ and $\mathbb{L}^{4}: \mathcal{H}_{h}$ and $\mathcal{H}_{\partial h}$ may possibly be singular and self-intersecting. Note that regularity assumptions on the support function $h$ can be weakened in many cases. For instance, to define the coenvelope $\mathcal{H}_{\partial h}$ in $\mathbb{L}^{4}$ we only need to assume that $h$ is of class $C^{2}$ on $\mathbb{S}^{2}$.

In everyday language, hedgehogs are spiny mammals. Note that Langevin, Levitt and Rosenberg chose to call $\mathcal{H}_{h}$ a hérisson [6], which is the French name for hedgehog, to illustrate the fact that, in each direction $u \in \mathbb{S}^{2}$, there is one and only one 'normal' $N_{h}(u):=x_{h}(u)+\mathbb{R} u$ pointing out from $\mathcal{H}_{h}$ (as, in each direction, a unique spine is pointing out from the skin of the spiny mammal). Similarly, it is worth pointing out, that, in each null direction $\left.u_{L}:=\frac{1}{\sqrt{2}}(u,-1)\right) \in\left(\mathbb{S}^{2} \times\{-1\}\right) / \sqrt{2} \subset \mathbb{S}^{3}$, there is one and only one null line $L_{h}(u):=x_{\partial h}(u)+\mathbb{R} u_{L}$ that is normal to the co-envelope $\mathcal{H}_{\partial h}$ in $\mathbb{L}^{4}$.

\subsubsection{Geometrical interpretation of co-hedgehogs of $\mathbb{L}^{4}$}

First, let us remind some basic facts about the curvature of hedgehogs of $\mathbb{R}^{3}$. Since the parametrization $x_{h}: \mathbb{S}^{2} \rightarrow \mathcal{H}_{h} \subset \mathbb{R}^{3}$ can be regarded as the inverse of the Gauss map, the Gauss curvature $K_{h}$ of $\mathcal{H}_{h}$ at $x_{h}(u)$ is given by $K_{h}(u)=$ $1 / \operatorname{det}\left[T_{u} x_{h}\right]$, where $T_{u} x_{h}$ is the tangent map of $x_{h}$ at $u$. Therefore, singularities of $\mathcal{H}_{h}$ are the points at which its Gauss curvature becomes infinite. For all $u \in \mathbb{S}^{2}$, the tangent map of $x_{h}$ at the point $u$ is $T_{u} x_{h}=h(u) I d_{T_{u} \mathbb{S}^{2}}+H_{h}^{u}$, where $H_{h}^{u}$ is the symmetric endomorphism associated with the Hessian of $h$ at $u$. Consequently, if $\lambda_{1}(u)$ and $\lambda_{2}(u)$ are the eigenvalues of the Hessian of $h$ at $u$ then $R_{1}(u):=\left(\lambda_{1}+h\right)(u)$ and $R_{2}(u):=\left(\lambda_{2}+h\right)(u)$ can be interpreted as the principal radii of curvature of $\mathcal{H}_{h}$ at $x_{h}(u)$, and the so-called curvature function $R_{h}:=1 / K_{h}$ is given by

$$
R_{h}(u)=\operatorname{det}\left[H_{i j}(u)+h(u) \delta_{i j}\right]=\left(R_{1} R_{2}\right)(u),
$$

where $\delta_{i j}$ are the Kronecker symbols and $\left(H_{i j}(u)\right)$ the Hessian of $h$ at $u$ with respect to an orthonormal frame on $\mathbb{S}^{2}$. In computations, it is often more convenient to replace $h$ by its positively 1 -homogeneous extension to $\mathbb{R}^{3} \backslash\{0\}$, which is given by,

$$
\varphi(x):=\|x\| h\left(\frac{x}{\|x\|}\right),
$$

for $x \in \mathbb{R}^{3} \backslash\{0\}$, where $\|\cdot\|$ is the Euclidean norm on $\mathbb{R}^{3}$. A straightforward computation gives:

(i) $x_{h}$ is the restriction of the gradient of $\varphi$ to the unit sphere $\mathbb{S}^{2}$;

(ii) For all $u \in \mathbb{S}^{2}$, the tangent map $T_{u} x_{h}$ identifies with the symmetric endomorphism associated with the Hessian of $\varphi$ at $u$.

In order to bring out our geometrical interpretation of the co-evolute $\mathcal{H}_{\partial h}$, 
we now give another expression for $x_{l_{h}}$. For all $u \in \mathbb{S}^{2}$, we have

$$
\begin{aligned}
x_{l_{h}}(u) \quad & :=l_{h}(u)+\delta(\partial h)(u) u_{L}=(\nabla h(u), h(u))+\frac{\Delta h(u)}{2}(u,-1) \\
& =\left(\nabla h(u)+\frac{\Delta h(u)}{2} u, h(u)-\frac{\Delta h(u)}{2}\right) \\
& =\left(x_{h}(u)-R_{(1, h)}(u) u, R_{(1, h)}(u)\right)=\left(c_{h}(u), R_{(1, h)}(u)\right),
\end{aligned}
$$

where $c_{h}(u)$ is the midpoint of the focal segment (which connects the two centers of principal curvatures of $\mathcal{H}_{h}$ at $\left.x_{h}(u)\right)$ and, $R_{(1, h)}:=h-\frac{\Delta h}{2}=\frac{1}{2}\left(R_{1}+R_{2}\right)$ is the mean radius of curvature of $\mathcal{H}_{h}$ (for the interpretation of $R_{(1, h)}$ as a mixed curvature function see e.g. [9]). Therefore, for all $u \in \mathbb{S}^{2}, x_{l_{h}}(u)$ can be interpreted geometrically as the (possibly reduced to a point) middle sphere of $\mathcal{H}_{h}$ at $x_{h}(u)$ that is cooriented by the vector $u$, which is normal to it, by identifying $\mathbb{L}^{4}$ to the so-called Laguerre space, say $\Sigma$, of cooriented spheres and (non-cooriented) point spheres of $\mathbb{R}^{3}$ via the bijection

$$
\begin{aligned}
& \mathbb{L}^{4} \rightarrow \Sigma \\
& (a, r) \mapsto S(a ; r),
\end{aligned}
$$

where $S(a ; r)$ denotes the sphere of radius $|r|$ centered at $a$ that is cooriented by its outward (resp. inward) pointing normal if $r>0$ (resp. $r<0$ ) holds, and the (non-cooriented) point sphere $\{a\}$ if $r=0$ holds. Thus:

Proposition 1. The co-evolute $\mathcal{H}_{\partial h}$ of $\mathcal{H}_{h}$ can be regarded as the locus of all the (possibly reduced to a point) cooriented middle spheres of $\mathcal{H}_{h} \subset \mathbb{R}^{3}$ in $\mathbb{L}^{4} \cong \Sigma$.

\subsubsection{Relationship with Laguerre geometry}

Laguerre geometry in $\mathbb{R}^{3}$ is based on oriented planes, cycles (i.e., oriented spheres and points regarded as unoriented spheres of radius zero) and oriented contact between them. The orientation is determined by a unit normal vector field or, equivalently, by a signed radius in the case of a sphere. An oriented sphere or an oriented hyperplane of $\mathbb{R}^{3}$ is said to be in oriented contact with another oriented sphere or hyperplane if they are tangent and moreover if their unit normals coincide at the point of tangency. An unoriented point sphere is said to be in oriented contact with an oriented sphere or hyperplane if it is contained in it. An affine Laguerre transformation of $\mathbb{L}^{4} \cong \Sigma$ is an affine transformation $\mathcal{A}(x):=\mathcal{L}(x)+\mathcal{C}$ of $\mathbb{L}^{4}$, where $L$ is a linear transformation that preserves the pseudo-scalar product $\langle., .\rangle_{L}$ (i.e., $\mathcal{L} \in O(3,1)$ ), and thus the tangential distance between spheres, and $\mathcal{C}$ is a vector of $\mathbb{L}^{4}$.

Classical references on sphere geometries of Laguerre and Lie are Blaschke's book [1] and, for a modern account, T. Cecil's book [2]. In these two references, 
it is shown that Laguerre geometry in $\mathbb{R}^{3}$ can be built as a subgeometry of Lie sphere geometry. The subgroup of 'Laguerre transformations' then consists of those Lie sphere transformations that map planes to planes. Each of these Laguerre transformations corresponds to an affine Laguerre transformation of $\mathbb{L}^{4}$ (as we have defined them above).

Laguerre geometry of surfaces studies properties and invariants of surfaces of $\mathbb{R}^{3}$ under the Laguerre transformation group. It has been extensively developed by Blaschke and its school [1]. Let us consider the case of hedgehogs of $\mathbb{R}^{3}$. Let $\Omega$ be an open domain of $\mathbb{S}^{2}$ such that $x_{h}: \Omega \rightarrow \mathbb{R}^{3}$ is an umbilic-free (piece of) hedgehog $\mathcal{H}_{h}$ with support function $h \in C^{\infty}(\Omega ; \mathbb{R})$. The geometric invariants of the (co)hedgehog $\mathcal{H}_{\partial h} \cong x_{l_{h}}(\Omega)$ of $\mathbb{L}^{4}$ are exactly the Laguerre invariants of the original hedgehog $\mathcal{H}_{h}$. The conformal immersion $x_{l_{h}}: \Omega \rightarrow \mathbb{L}^{4}$, which we consider as a natural parametrization $x_{\partial h}: \Omega \rightarrow \mathbb{L}^{4}$ of the co-evolute $\mathcal{H}_{\partial h}$ of $\mathcal{H}_{h}$, assigns to each $u \in \Omega$ the point of $\mathbb{L}^{4}$ corresponding to the oriented middle sphere or unoriented middle point $S\left(c_{h}(u) ; R_{(1, h)}(u)\right) \in \mathbb{L}^{4} \cong \Sigma$ that is in oriented contact with the oriented support plane of $\mathcal{H}_{h}$ at $x_{h}(u)$. It will be called the Laguerre Gauss map of $x_{h}: \Omega \rightarrow \mathbb{R}^{3}$.

Note that the set of marginally trapped hedgehogs $\mathcal{H}_{\partial h} \cong x_{l_{h}}\left(\mathbb{S}^{2}\right)$ of $\mathbb{L}^{4}$, identified with their support differentials $\partial h:=d h / \sqrt{2}$, constitute a real linear space, say $\partial \mathcal{H}$. The surface area of marginally trapped hedgehogs of $\mathbb{L}^{4}$ defines a particularly interesting Laguerre invariant functional on $\partial \mathcal{H}$, the so-called Laguerre functional:

$$
\begin{aligned}
L: \quad \partial \mathcal{H} & \rightarrow \mathbb{R} \\
\partial h & \mapsto L(\partial h):=\frac{1}{4} \int_{\mathbb{S}^{2}}\left(R_{1}-R_{2}\right)^{2} d \sigma=\int_{\mathbb{S}^{2}}\left(R_{(1, h)}^{2}-R_{h}\right) d \sigma,
\end{aligned}
$$

where $\sigma$ is the spherical Lebesgue measure on $\mathbb{S}^{2}, R_{(1, h)}$ the mean radius of curvature, and $R_{h}$ the curvature function of $\mathcal{H}_{h}$. For any $h \in C^{\infty}(\Omega ; \mathbb{R}), L(\partial h)$ will be called the Laguerre area of $\mathcal{H}_{h}$ and will sometimes be denoted by $L\left(x_{h}\right)$.

The surface area element of $\mathcal{H}_{\partial h}$ can be interpreted in $\mathbb{R}^{3}$ by considering the mean surface of $\mathcal{H}_{h}$, say $\mathcal{M}_{h}$, which we define as the (possibly singular and self-intersecting) surface that is parametrized by $c_{h}: \mathbb{S}^{2} \rightarrow \mathbb{R}^{3}, u \mapsto c_{h}(u)$. Indeed, if we denote by $d \mu_{h}(u)$ the corresponding surface area element of this mean surface $\mathcal{M}_{h}$, then it is pure routine to check that:

$\left(R_{(1, h)}^{2}-R_{h}\right)(u) d \sigma(u)$ can be regarded as the orthogonal projection of $d \mu_{h}(u)$ into the plane that is parallel to the support plane $P_{h}(u)$ of $\mathcal{H}_{h}$ and passes through $c_{h}(u) \in \mathcal{M}_{h}$.

The following theorem is a first step towards a 'Brunn-Minkowski theory' for marginally trapped hedgehogs, which will be the topic of our next subsection.

Theorem 2. The map $\sqrt{L}: \partial \mathcal{H} \rightarrow \mathbb{R}_{+}, \partial h \longmapsto \sqrt{L(\partial h)}$ is a norm associated with the scalar product 


$$
\begin{aligned}
L: \quad(\partial \mathcal{H})^{2} & \mapsto \mathbb{R}, \\
(\partial f, \partial g) & \longmapsto L(\partial f, \partial g):=\int_{\mathbb{S}^{2}}\left(R_{(1, f)} R_{(1, g)}-R_{(f, g)}\right) d \sigma,
\end{aligned}
$$

where $R_{(. . .)}$denotes the mixed curvature function of hedgehogs (see e.g. [9] for a detailed definition and fundamental properties of $\left.R_{(. . .)}\right)$.

In particular, for all $(\partial f, \partial g) \in(\partial \mathcal{H})^{2}$, we have

$$
\sqrt{L(\partial f+\partial g)} \leq \sqrt{L(\partial f)}+\sqrt{L(\partial g)},
$$

and

$$
L(\partial f, \partial g)^{2} \leq L(\partial f) L(\partial g),
$$

with equalities if, and only if, $\mathcal{H}_{\partial f}$ and $\mathcal{H}_{\partial g}$ are homothetic (here, "homothetic" means that there exists $(\lambda, \mu) \in \mathbb{R}^{2}-\{(0,0)\}$ such that $\left.\lambda \partial f+\mu \partial g=0\right)$.

The scalar product $L(\partial f, \partial g)$ will of course be called the mixed Laguerre area of $\mathcal{H}_{\partial f}$ and $\mathcal{H}_{\partial g}$, and will sometimes be denoted by $L\left(x_{f}, x_{g}\right)$.

We will return to the Laguerre geometry of hedgehogs in the framework of contact geometry.

\subsection{Relationship with the Brunn-Minkowski theory}

Classical hedgehog theory is an extension of the Brunn-Minkowski theory. The relationship between hedgehogs and marginally trapped surfaces is thus very promising in terms of new geometric inequalities. We already mentioned two fundamental geometric inequalities for marginally trapped hedgehogs in our Theorem 2. Here, it is worth pointing out how important geometric inequalities are in gravitation since they provide information on the relationship between physically relevant magnitudes in a robust way. We will see that marginally trapped hedgehogs arise naturally in a 3-dimensional equivalent of a classical geometric inequality for hedgehogs of the Euclidean plane.

Classical isoperimetric inequalities involving evolutes for hedgehog curves are involving (co)evolutes for hedgehog surfaces

In [8] the author already introduced $\mathcal{H}_{\partial h}$ as a hedgehog with support differential $\partial h:=d h / \sqrt{2}$ but without precisely grasping the essence of the co-contact structure. Let us recall how marginally trapped hedgehogs appeared in order to play the role of evolutes in a natural 3-dimensional equivalent of a known upper bound of the isoperimetric deficit of plane hedgehog curves in terms of signed area of their evolute.

H. Geppert was the first to introduce hedgehogs in $\mathbb{R}^{2}$ and $\mathbb{R}^{3}$ (under the German names stützbare Bereiche and stützbare Flächen) in 1937 [5] in an attempt 
to extend parts of the Brunn-Minkowski theory. Many classical inequalities for convex curves find their counterparts for hedgehogs. Of course, adaptations are necessary. In particular, lengths and areas have to be replaced by algebraic versions. For instance, Theorem 3 below extends the isoperimetric inequality $L^{2}-4 \pi A \geq 0$, which holds for any planar convex body $K$ with perimeter $L$, and area $A$, to any hedgehog $\mathcal{H}_{h}$ of the Euclidean plane $\mathbb{R}^{2}$, and gives an upper bound of the isoperimetric deficit in terms of signed area of the evolute.

Theorem 3 ([7, Prop. 6, Upper bound of the isoperimetric deficit in terms of signed area of the evolute]). For any $h \in C^{4}\left(\mathbb{S}^{1} ; \mathbb{R}\right)$, let $\mathcal{H}_{h}$ be the hedgehog of $\mathbb{R}^{2}$ with support function $h$ (i.e. the envelope of the family of cooriented lines with equation $\langle x, u(\theta)\rangle=h(\theta)$, where $u(\theta)=(\cos \theta, \sin \theta))$. We have:

$$
0 \leq l(h)^{2}-4 \pi a(h) \leq-4 \pi a(\partial h)=-4 \pi a\left(h, \partial^{2} h\right)
$$

where $l(h)$ is the length of $\mathcal{H}_{h}, a(h)$ its area, $\partial h(\theta):=h^{\prime}\left(\theta-\frac{\pi}{2}\right)$ the support function of its evolute $\mathcal{H}_{\partial h}, \partial^{2} h:=-h^{\prime \prime}$ the one of its second evolute $\mathcal{H}_{\partial^{2} h}$, and $a\left(h, \partial^{2} h\right)$ the mixed area of $\mathcal{H}_{h}$ and $\mathcal{H}_{\partial^{2} h}$. In each inequality of (I), the equality holds if and only if $\mathcal{H}_{h}$ is a circle or a point.

The isoperimetric inequality $L^{2}-4 \pi A \geq 0$ admits the following 3-dimensional equivalent. Given a convex body $K$ in the Euclidean 3 -space $\mathbb{R}^{3}$, with surface area $S$ and integral of the mean curvature $M$, the first Minkowski inequality for mixed volumes ensures that

$$
M^{2}-4 \pi S \geq 0
$$

where equality holds if and only if $K$ is a ball (see e.g. [13] ). In [8] the author proved the following 3-dimensional equivalent of Theorem 3:

Theorem 4 ([8, Theorem 1, Upper bound of the 'deficit' $M^{2}-4 \pi S$ in terms of signed area of the co-evolute surface] ). For any $h \in C^{4}\left(\mathbb{S}^{2} ; \mathbb{R}\right)$, let $\mathcal{H}_{h}$ denote the hedgehog of $\mathbb{R}^{3}$ with support function $h$, (i.e. the envelope of the family of cooriented planes with equation $\langle x, u\rangle=h(u)$ in $\left.\mathbb{R}^{3}\right)$. We have:

$$
0 \leq m(h)^{2}-4 \pi s(h) \leq-4 \pi s(\partial h)=-4 \pi s\left(h, \partial^{2} h\right),
$$

where $m(h)$ is the integral of the mean curvature of $\mathcal{H}_{h}, s(h)$ its signed surface area, $s(\partial h)$ the signed surface area of $\mathcal{H}_{\partial h}$, and $s\left(h, \partial^{2} h\right)$ the mixed surface area of $\mathcal{H}_{h}$ and of its mean evolute $\mathcal{H}_{\partial^{2} h}$. In each inequality of (II), the equality holds if and only if $\mathcal{H}_{h}$ is a sphere or a point.

Here, the signed surface area $s(\partial h)$ is the opposite of the Laguerre area $L(\partial h)$, which we have introduced in the previous subsection. In other words, $s(\partial h)=-L(\partial h)$. Besides, $\sqrt{2} \partial$ stands for the Hodge-Dirac operator $D=d+\delta$ on $\mathbb{S}^{2}$, where $d$ is the exterior differentiation and $\delta=-* d *$ the codifferential, so that $\partial h=d h / \sqrt{2}$ and $\partial^{2} h=\frac{1}{2} \delta(d h)=\frac{1}{2} \Delta h(u)$. Recall that the mean evolute 
of $\mathcal{H}_{h}$ is the envelope of the family of planes parallel to the support planes to $\mathcal{H}_{h}$ and passing through the midpoints of the focal segments (which connect the two centers of principal curvature of $\mathcal{H}_{h}$ in $\mathbb{R}^{3}$ ). Now in the planar case, $\partial$ can be interpreted as the Hodge-Dirac operator $D=d+\delta$ on $\mathbb{S}^{1}$. Indeed, $d h_{\theta}=h^{\prime}(\theta) d \theta$ can be interpreted as the support function $\partial h(\theta)$ of the evolute $\mathcal{H}_{\partial h}$ in the sense that $\mathcal{H}_{\partial h}$ is the envelope of the family of cooriented lines with equation $\left\langle x, u^{\prime}(\theta)\right\rangle=h^{\prime}(\theta)$, and $D^{2} h=-h^{\prime \prime}$. Thus, in this equivalence between these two isometric inequalities (for hedgehog curves and surfaces), we have the following correspondences:

\begin{tabular}{|c|c|}
\hline $\begin{array}{c}\text { Hedgehog curve } \mathcal{H}_{h} \text { in } \mathbb{R}^{2} \\
\downarrow \partial\end{array}$ & $\begin{array}{c}\text { Hedgehog surface } \mathcal{H}_{h} \text { in } \mathbb{R}^{3} \\
\downarrow \partial \\
\text { Evolute curve } \mathcal{H}_{\partial h} \text { in } \mathbb{R}^{2} \\
\downarrow \partial\end{array}$ \\
$\begin{array}{c}\text { Co-evolute surface of } \mathcal{H}_{h} \text { in } \mathbb{L}^{4} \\
\text { (Marginally trapped hedgehog } \mathcal{H}_{\partial h}\end{array}$ \\
$\downarrow \partial$ \\
Second evolute curve $\mathcal{H}_{\partial^{2} h}$ in $\mathbb{R}^{2}$ & Mean evolute surface $\mathcal{H}_{\partial^{2} h}$ in $\mathbb{R}^{3}$ \\
\hline
\end{tabular}

where $\sqrt{n} \partial$ denote the Hodge-Dirac operator $D=d+\delta$ on $\mathbb{S}^{n}$, with respectively $n=1$ and 2 for curves and surfaces.

Remark in the 3-dimensional case. For any $h \in C^{4}\left(\mathbb{S}^{2} ; \mathbb{R}\right)$, we have the following commutative diagram:

\footnotetext{
Transition to (cooriented) middle spheres-points of $\mathcal{H}_{h}$

$$
\mathcal{H}_{\partial h} \subset \mathbb{L}^{4} \cong \Sigma
$$$$
\mathcal{H}_{h} \subset \mathbb{R}^{3}
$$

Transition to

the mean evolute$$
\mathcal{H}_{\partial^{2} h} \subset \mathbb{R}^{3}
$$

Passage over into the spacetime and reincarnation in $\mathbb{R}^{3}$. The marginally trapped hedgehog $\mathcal{H}_{\partial h}$ can be thought as the 'Hodge-Dirac evolute' of $\mathcal{H}_{h}$. This evolute therefore lives in $\mathbb{L}^{4}$ (and not in $\mathbb{R}^{3}$ ) whereas the mean evolute of $\mathcal{H}_{h}$ (which can be thought as the 'second Hodge-Dirac evolute' $\mathcal{H}_{\partial^{2} h}$ of $\mathcal{H}_{h}$, or equivalently, as the 'first Hodge-Dirac evolute' of $\mathcal{H}_{\partial h}$ ) does live in $\mathbb{R}^{3}$. For any
}

Transition to the envelope in $\mathbb{R}^{3}$ of $(P(u))_{u \in \mathbb{S}^{2}}$, where $P(u)$ is the plane through the center of the sphere $x_{\partial h}(u)$ that is cooriented by $u,\left(u \in \mathbb{S}^{2}\right)$ 
$h \in C^{\infty}\left(\mathbb{S}^{2} ; \mathbb{R}\right)$, we can thus consider the sequence $\left(\mathcal{H}_{\partial^{n} h}\right)_{n \in \mathbb{N}}$ of 'hedgehogs', which is such that:

(i) for any even $n=2 k,\left(k \in \mathbb{N}^{*}\right), \mathcal{H}_{\partial^{2 k} h}$ is the (ordinary) hedgehog of $\mathbb{R}^{3}$ that is the mean evolute of $\mathcal{H}_{\partial^{2(k-1)} h} \subset \mathbb{R}^{3}$;

(ii) for any odd $n=2 k+1,(k \in \mathbb{N}), \mathcal{H}_{\partial^{2 k+1} h}$ is the (marginally trapped) hedgehog of $\mathbb{L}^{4}$ that is the co-evolute of $\mathcal{H}_{\partial^{2 k} h} \subset \mathbb{R}^{3}$.

The support vector field of $\mathcal{H}_{\partial h}$. For any $h \in C^{4}\left(\mathbb{S}^{2} ; \mathbb{R}\right)$, let $\overrightarrow{\partial h}$ denote the vector field that corresponds to the 1 -form $\partial h:=d h / \sqrt{2}$ by the canonical musical duality:

$$
\forall \vec{X} \in T \mathbb{S}^{2}, \quad(\partial h)(\vec{X})=g(\overrightarrow{\partial h}, X)=\overrightarrow{\partial h} \cdot \vec{X},
$$

where $g:(\vec{X}, \vec{Y}) \mapsto g(\vec{X}, \vec{Y})=\vec{X} \cdot \vec{Y}$ denotes the standard metric on $\mathbb{S}^{2}$.

\section{Similarity of formulas for hedgehogs and (co)hedgehogs}

Theorem 5. For any $h \in C^{4}\left(\mathbb{S}^{2} ; \mathbb{R}\right)$, the signed surface area $s(\partial h)$ of $\mathcal{H}_{\partial h}$ can be expressed in the following forms:

$$
s(\partial h)=\int_{\mathbb{S}^{2}}\left((\overrightarrow{\partial h})^{2}-\left(\partial^{2} h\right)^{2}\right) d \sigma=\int_{\mathbb{S}^{2}} \overrightarrow{\partial h} \cdot \overrightarrow{\partial R_{(1, h)}} d \sigma,
$$

where $\sigma$ is the spherical Lebesgue measure on $\mathbb{S}^{2}, \partial^{2} h$ the support function of the mean evolute of $\mathcal{H}_{h}$, and $R_{(1, h)}$ the mean radius of curvature of $\mathcal{H}_{h}$.

It is worth noting the similarity with the known formulas for the signed surface area of a hedgehog $\mathcal{H}_{h}$ of $\mathbb{R}^{3}$ :

$$
s(h)=\int_{\mathbb{S}^{2}}\left(h^{2}-(\overrightarrow{\partial h})^{2}\right) d \sigma=\int_{\mathbb{S}^{2}} h \cdot R_{(1, h)} d \sigma,
$$

where $h \in C^{2}\left(\mathbb{S}^{2} ; \mathbb{R}\right)$.

Let us recall the proof of these two last inequalities. From the definition

$$
s(h)=\int_{\mathbb{S}^{2}} R_{h} d \sigma,
$$

we obtain $s(h)=\int_{\mathbb{S}^{2}} h \cdot R_{(1, h)} d \sigma$ by the symmetry of the mixed volume (see e.g. $[7])$, and then $s(h)=\int_{\mathbb{S}^{2}}\left(h^{2}-(\overrightarrow{\partial h})^{2}\right) d \sigma$ by integrating by parts.

In the same vein as Theorem 5 , we can notice that the signed surface area of the mean evolute $\mathcal{H}_{\partial^{2} h}$ of $\mathcal{H}_{h}$ is given by:

$$
s\left(\partial^{2} h\right)=\int_{\mathbb{S}^{2}}\left(\left(\partial^{2} h\right)^{2}-\overrightarrow{\partial\left(\partial^{2} h\right)^{2}}\right) d \sigma=\int_{\mathbb{S}^{2}}\left(\partial^{2} h\right) \cdot \partial^{2} R_{(1, h)} d \sigma .
$$


From the above formulas, we deduce that the mixed surface area of two hedgehogs $\mathcal{H}_{f}$ and $\mathcal{H}_{g}$ of $\mathbb{R}^{3}$ is given by:

$$
s(f, g)=\int_{\mathbb{S}^{2}}(f g-\overrightarrow{\partial f} \cdot \overrightarrow{\partial g}) d \sigma=\int_{\mathbb{S}^{2}} f \cdot R_{(1, g)} d \sigma=\int_{\mathbb{S}^{2}} g \cdot R_{(1, f)} d \sigma .
$$

Now, the integration by parts formula can be written as

$$
\int_{\mathbb{S}^{2}} \overrightarrow{\partial f} \cdot \overrightarrow{\partial g} d \sigma=\int_{\mathbb{S}^{2}} f \cdot \partial^{2} g d \sigma
$$

for all $(f, g) \in C^{4}\left(\mathbb{S}^{1} ; \mathbb{R}\right)^{2}$. Therefore

$$
\int_{\mathbb{S}^{2}} \overrightarrow{\partial f} \cdot \overrightarrow{\partial R_{(1, g)}} d \sigma=\int_{\mathbb{S}^{2}} f \cdot \partial^{2} R_{(1, g)} d \sigma=\int_{\mathbb{S}^{2}} f \cdot R_{\left(1, \partial^{2} g\right)} d \sigma=s\left(f, \partial^{2} g\right),
$$

and so, by the symmetry of the mixed surface area of hedgehogs,

$$
\int_{\mathbb{S}^{2}} \overrightarrow{\partial f} \cdot \overrightarrow{\partial R_{(1, g)}} d \sigma=s\left(\partial^{2} g, f\right)=\int_{\mathbb{S}^{2}} \partial^{2} g \cdot R_{(1, f)} d \sigma,
$$

and again by the integration by parts formula

$$
\int_{\mathbb{S}^{2}} \overrightarrow{\partial f} \cdot \overrightarrow{\partial R_{(1, g)}} d \sigma=\int_{\mathbb{S}^{2}} \overrightarrow{\partial g} \cdot \overrightarrow{\partial R_{(1, f)}} d \sigma
$$

Thus, we have deduced the following corollary to Theorem 5 .

Corollary 1. For any $(f, g) \in C^{4}\left(\mathbb{S}^{2} ; \mathbb{R}\right)^{2}$, let $s(\partial f, \partial g)$ denote the mixed surface area of $\mathcal{H}_{\partial f}$ and $\mathcal{H}_{\partial g}$, that is $s(\partial f, \partial g):=-L(\partial f, \partial g)$. This mixed area $s(\partial f, \partial g)$ can be expressed in the following forms:

$$
s(\partial f, \partial g)=\int_{\mathbb{S}^{2}} \overrightarrow{\partial f} \cdot \overrightarrow{\partial R_{(1, g)}} d \sigma=\int_{\mathbb{S}^{2}} \overrightarrow{\partial g} \cdot \overrightarrow{\partial R_{(1, f)}} d \sigma=s\left(f, \partial^{2} g\right)=s\left(\partial^{2} f, g\right),
$$

where $\sigma$ is the spherical Lebesgue measure on $\mathbb{S}^{2}$.

In particular, the mixed surface area of the marginally trapped hedgehogs $\mathcal{H}_{\partial f}$ and $\mathcal{H}_{\partial g}$ is equal to the mixed surface area of the (ordinary) hedgehogs $\mathcal{H}_{f}$ and $\mathcal{H}_{\partial^{2} g}$, which is the mean evolute of $\mathcal{H}_{g}$.

\section{Hedgehog theory is not restricted to Euclidean spaces}

Of course, the classical hedgehog theory is not restricted to Euclidean spaces. In [4], F. Fillastre introduced and studied 'Fuchsian convex bodies', which are the closed convex sets of the Lorentz-Minkowski space $\mathbb{L}^{n+1}$ that are globally invariant under the action of some Fuchsian group. In particular, he gave a 
'reversed Alexandrov-Fenchel inequality' and thus a 'reversed Brunn-Minkowski inequality'. In [11], the author gave a detailed study of plane Lorentzian and Fuchsian hedgehogs and also proved a series of Fuchsian analogues of classical geometrical inequalities (which are also reversed as compared to classical ones).

\subsection{Synthetic and comparative co-presentation of hedge- hogs and (co)hedgehogs}

In this section, we will now compare carefully the definitions of hedgehogs in $\mathbb{R}^{3}$ and of (co)hedgehogs (i.e., marginally trapped hedgehogs) in $\mathbb{L}^{4}$, and then for the convenience of the reader, we will summarize schematically our comparison in tables.

As recalled in Subsection 1.1, the datum of any $h \in C^{\infty}\left(\mathbb{S}^{2} ; \mathbb{R}\right)$ determines the hedgehog $\mathcal{H}_{h}$ in $\mathbb{R}^{3}$ as the envelope of the family $\left(P_{h}(u)\right)_{u \in \mathbb{S}^{2}}$ of cooriented planes with equation $\langle x, u\rangle=h(u),\left(u \in \mathbb{S}^{2}\right)$. Analytically, taking the envelope of $\mathcal{P}$ amounts to solving the following system of equations

$$
\left\{\begin{array}{l}
\langle x, u\rangle=h(u) \\
\langle x, d u\rangle=d h_{u}
\end{array}\right.
$$

for all $u \in \mathbb{S}^{2}$, where the second equation is deduced from the first via the contact condition $\langle d x, u\rangle=0$ (and thus by performing a partial differentiation with respect to $u)$.

Now, as we saw in Subsection 1.1, the datum of $\partial h:=d h / \sqrt{2}$ determines the (co)hedgehog (i.e., marginally trapped hedgehog) $\mathcal{H}_{\partial h}$ in $\mathbb{L}^{4}$ as the co-envelope of the family $\left(L_{h+t}(u)\right)_{(u, t) \in \mathbb{S}^{2} \times \mathbb{R}}$ of oriented null lines defined by $L_{h+t}(u):=$ $\{(\nabla h(u), h(u)+t)\}+\mathbb{R} u_{L}$. Analytically, taking the co-envelope of $\mathcal{L}$ amounts to solving the following system of equations

$$
(S)\left\{\begin{array}{l}
\left\{\begin{array}{l}
\left\langle x, u_{L}\right\rangle_{L}=(h(u)+t) / \sqrt{2} \\
\left\langle x, d u_{L}\right\rangle_{L}=\partial h(u)
\end{array}\right. \\
\left\langle x, \Delta u_{L}\right\rangle_{L}=\delta(d h)(u)
\end{array}\right.
$$

for all $(u, t) \in \mathbb{S}^{2} \times \mathbb{R}$, where the third equation is deduced from the two first ones via the co-contact condition $\left\langle\Delta x, u_{L}\right\rangle_{L}=0$ (or, equivalently, by performing a partial codifferentiation with respect to $u$ in the second equation). Here, the Hodge-Laplace operator of a vector function $X: \mathbb{S}^{2} \rightarrow \mathbb{L}^{4}, u \mapsto\left(X_{i}(u)\right)_{i=1}^{4}$ is of course understood to be the vector function $\Delta X: \mathbb{S}^{2} \rightarrow \mathbb{L}^{4}, u \mapsto\left(\Delta X_{i}(u)\right)_{i=1}^{4}$, where $\Delta X_{i}$ is the Hodge-Laplace operator of the coordinate function $X_{i}$ in the intrinsic metric on $\mathbb{S}^{2},(1 \leq i \leq 4)$. Note that the system formed by the two first equations of $(S)$ simply traduces the fact that $x \in L_{h+t}(u)$. The second equation can be deduced from the first by using the condition $\left\langle d x, u_{L}\right\rangle_{L}=0$ (and thus by performing a partial differentiation with respect to $u$ ).

For any $h \in C^{\infty}\left(\mathbb{S}^{2} ; \mathbb{R}\right)$, we therefore have the following comparison table between both definitions: 


\begin{tabular}{|c|c|}
\hline $\begin{array}{l}\text { The hedgehog } \mathcal{H}_{h} \text { can be defined } \\
\text { in } \mathbb{R}^{3} \text { as the envelope of the family } \\
\left(P_{h}(u)\right)_{u \in \mathbb{S}^{2}} \text { of cooriented planes } \\
\text { with equation }\langle x, u\rangle=h(u) \text {. }\end{array}$ & $\begin{array}{l}\text { The (co)hedgehog } \mathcal{H}_{\partial h} \text { can be defined } \\
\text { in } \mathbb{L}^{4} \text { as the co-envelope of the family } \\
\left(L_{h+t}(u)\right)_{(u, t) \in \mathbb{S}^{2} \times \mathbb{R}} \text { of oriented null lines } \\
\text { defined by : } \\
L_{h+t}(u):=\{(\nabla h(u), h(u)+t)\}+\mathbb{R} u_{L}\end{array}$ \\
\hline $\begin{array}{l}\text { (1) } \quad\langle x, u\rangle=h(u) \\
\quad[\text { via the contact condition }\end{array}$ & (1) $\left\{\begin{array}{cc}(1 . a) \quad\left\langle x, u_{L}\right\rangle_{L}=(h(u)+t) / \sqrt{2} \\
\Downarrow \quad\left(\text { via }\left\langle d x, u_{L}\right\rangle_{L}=0 \text { or a partial }\right. \\
\text { differentiation with respect to } u)\end{array}\right.$ \\
\hline$\Downarrow \quad(d) \quad\langle d x, u\rangle=0$ & $(1 . b) \quad\left\langle x, d u_{L}\right\rangle_{L}=\partial h(u)$ \\
\hline $\begin{array}{l}\text { or a partial differentiation } \\
\text { with respect to } u] \\
\text { (2) }\langle x, d u\rangle=d h_{u}\end{array}$ & $\begin{array}{l}\quad(\text { via the co-contact condition } \\
\Downarrow \quad(\delta) \quad\left\langle\delta(d x), u_{L}\right\rangle_{L}=0, \\
\text { or a codifferentiation with respect to } u) \\
\text { (2) }\left\langle x, \Delta u_{L}\right\rangle=\delta(d h)(u)\end{array}$ \\
\hline $\begin{array}{l}\text { For every } u \in \mathbb{S}^{2} \text {, the system } \\
\qquad \begin{cases}(1) & \langle x, u\rangle=h(u) \\
(2) & \langle x, d u\rangle=d h_{u},\end{cases} \\
\text { implies } x=h(u) u+\nabla h(u)\end{array}$ & $\begin{array}{l}\text { For every }(u, t) \in \mathbb{S}^{2} \times \mathbb{R} \text {, the system } \\
\left\{\begin{array}{l}(1)=(1 . a) \text { and }(1 . b): \quad x \in L_{h+t}(u) \\
(2) \quad\left\langle x, \Delta u_{L}\right\rangle=\delta(d h)(u)\end{array}\right. \\
\text { implies } x=(\nabla h(u), h(u)+t)+\delta(\partial h)(u) u_{L}\end{array}$ \\
\hline $\begin{array}{l}\text { In the absence of singularities, } \\
x_{h}: \mathbb{S}^{2} \rightarrow \mathcal{H}_{h}, u \mapsto h(u) u+\nabla h(u) \\
\text { can be interpreted as the inverse } \\
\text { of the Gauss map of } \mathcal{H}_{h} .\end{array}$ & $\begin{array}{l}\text { In the absence of umbilical points, } \\
x_{\partial h}: \Omega \rightarrow \mathcal{H}_{\partial h}, u \mapsto l_{h}(u)+\delta(\partial h)(u) u_{L} \\
\text { can be interpreted as the Laguerre Gauss } \\
\text { map of } x_{h}: \Omega \rightarrow \mathbb{R}^{3} \text {. }\end{array}$ \\
\hline
\end{tabular}

The cooriented support plane $P_{h}(u)$ is determined by $h(u) u$ and thus by the value of the support function $h$ at $u$, and $x_{h}(u)$ is then determined on $P_{h}(u)$ by the contact condition which imposes $x_{h}(u)-h(u) u=\nabla h(u)$. Analogously, the oriented support null line $L_{h}(u)$ is determined by $l_{h}(u)$ and thus by the value of the support differential $\partial h$ at $u$, and $x_{\partial h}(u)$ is then determined on $L_{h}(u)$ by the co-contact condition which imposes $x_{\partial h}(u)-l_{h}(u)=\delta(\partial h)(u) u_{L}$ : 


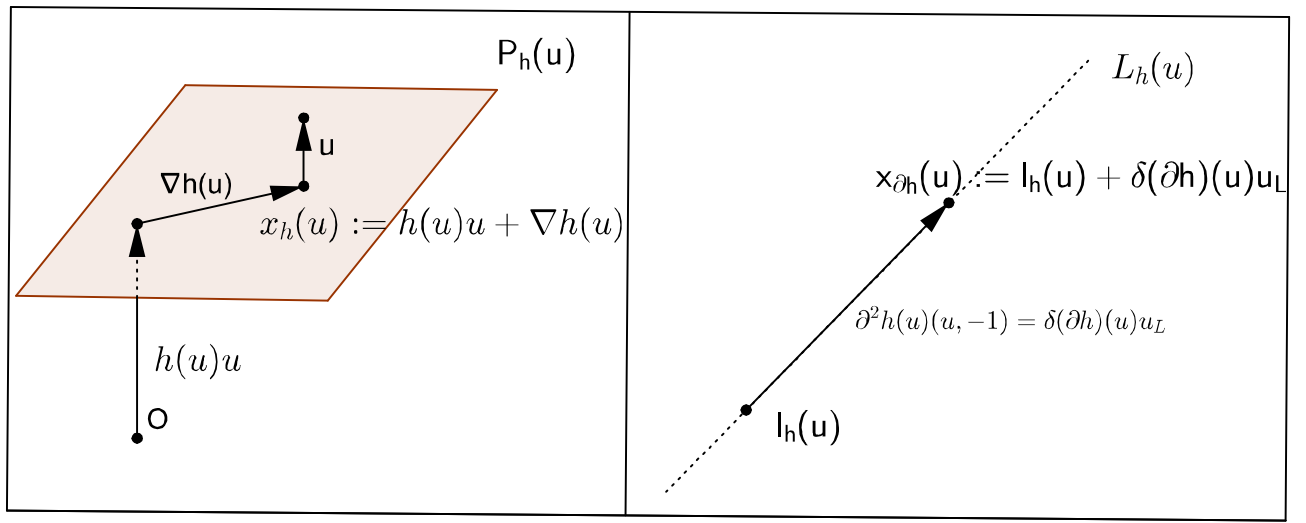

The role of $(d)$ and $(\delta)$ in the determination of $\mathcal{H}_{h}$ and $\mathcal{H}_{\partial h}$

The contact condition $(d)$ allows us to write

$$
\langle x, d u\rangle=d(\langle x, u\rangle) .
$$

The co-contact condition $(\delta)$ allows us to write $\quad\left\langle x, \Delta u_{L}\right\rangle_{L}=\Delta\left(\left\langle x, u_{L}\right\rangle_{L}\right)$ provided that $\quad\left\langle d x, u_{L}\right\rangle=0$.

What is meant by the contact and co-contact conditions $(d)$ and $(\delta)$

Recall first that, for every $C^{2}$-map $x: \mathbb{S}^{2} \rightarrow \mathbb{L}^{4}$ and every $u \in \mathbb{S}^{2}, \Delta x(u)=$ $\delta(d x)(u)$ can be interpreted as a second order derivative that measures, both in direction and magnitude, how $x(u)$ deviates from the average of $x$ over an infinitesimal sphere centered at $u$ in $\mathbb{S}^{2}$.

\begin{tabular}{|l|c|}
\hline Condition $(d) \quad\langle d x, u\rangle=0$ on $x: \mathbb{S}^{2} \rightarrow \mathbb{R}^{3}$ & Condition $(\delta) \quad\left\langle\delta(d x), u_{L}\right\rangle_{L}=0$ on $x: \mathbb{S}^{2} \rightarrow \mathbb{L}^{4}$ \\
\hline For all $u \in \mathbb{S}^{2}, u$ is orthogonal to the & For all $u \in \mathbb{S}^{2}, u_{L}$ is orthogonal in $\left(\mathbb{L}^{4},\langle., .\rangle_{L}\right)$ \\
tangent space $T_{x(u)} x\left(\mathbb{S}^{2}\right)=\left(T_{u} x\right)\left(T_{u} \mathbb{S}^{2}\right)$ & to the above mentioned measure of deviation \\
in the Euclidean space $\left(\mathbb{R}^{3},\langle.,\rangle.\right)$. & (in direction and magnitude) $\Delta x(u)=\delta(d x)(u)$. \\
\hline
\end{tabular}

\section{Marginally trapped hedgehogs in other spaces and generalizations}

\subsection{Marginally trapped hedgehogs or co-hedgehogs in $\mathrm{M}^{4}$}

\subsubsection{Characterization and definitions in $\mathrm{M}^{4}$}

As already mentioned, the hedgehog theory is not restricted to Euclidean spaces. For instance, in our preliminary study of Subsection 1.1 , we can replace $\left(\mathbb{R}^{3},\langle.,\rangle.\right)$ by the Lorentzian-Minkowski 3 -space $\mathbb{L}^{3}=\left(\mathbb{R}^{3},\langle., .\rangle_{L}\right)$, where

$$
\left\langle x, x^{\prime}\right\rangle_{L}=x_{1} x_{1}^{\prime}+x_{2} x_{2}^{\prime}-x_{3} x_{3}^{\prime},
$$


for all $x:=\left(x_{1}, x_{2}, x_{3}\right)$ and $x^{\prime}:=\left(x_{1}^{\prime} x_{2}^{\prime}, x_{3}^{\prime}\right)$ in $\mathbb{R}^{3}$, and the unit sphere $\mathbb{S}^{2}$ of $\mathbb{R}^{3}$ by the 'unit sphere'

$$
\mathbf{H}^{2}:=\left\{x=\left(x_{1}, x_{2}, x_{3}\right) \in \mathbb{L}^{3} \mid\langle x, x\rangle_{L}=-1\right\},
$$

which is a two-sheeted hyperboloid with constant Gaussian curvature -1 with respect to the induced metric. To any $h \in C^{\infty}\left(\mathbf{H}^{2} ; \mathbb{R}\right)$ corresponds the envelope $\mathcal{H}_{h}$ of the family $\left(P_{h}(v)\right)_{v \in \mathbb{S}^{2}}$ of cooriented spacelike planes of $\mathbb{L}^{3}$ with equation (E) $\langle x, v\rangle_{L}=h(v)$. We say that $\mathcal{H}_{h}$ is the hedgehog of $\mathbb{L}^{3}$ with support function $h$. From $(E)$ and the contact condition $\langle d x, v\rangle_{L}=0$, we deduce

$$
\left\{\begin{array}{l}
\langle x, v\rangle_{L}=h(v) \\
\langle x, d v\rangle_{L}=d h_{v}
\end{array}\right.
$$

for all $v \in \mathbf{H}^{2}$. Thus, it appears that $\mathcal{H}_{h}$ can be parametrized by $x_{h}: \mathbf{H}^{2} \rightarrow \mathbb{L}^{3}$, $v \mapsto(\nabla h)(v)-h(v) v$, where $(\nabla h)(v)$ stands for the gradient of $h$ at $v$. The parametrization $x_{h}$ can be interpreted as the inverse of its Gauss map (if $v$ is a regular point of $x_{h}$, then $v$ is normal to $\mathcal{H}_{h}$ at $\left.x_{h}(v)\right)$. Note that $\left(\mathcal{H}_{h+t}\right)_{t \in \mathbb{R}}$ is a family of parallel hedgehogs in $\mathbb{R}^{3}$ : for all $(v, t) \in \mathbf{H}^{2} \times \mathbb{R}, x_{h+t}(v)=x_{h}(v)-t v$.

Now, we claim that:

To the differential dh of $h$ corresponds naturally a 'marginally trapped hedgehog' of the Lorentz Minkowski 4-space $\mathbf{M}^{4}=\left(\mathbb{R}^{4},\langle., .\rangle_{M}\right)$ via a 'lightlike co-contact condition'.

Here, the pseudo-scalar product $\langle., .\rangle_{M}$ is defined by

$$
\left\langle(x, t),\left(x^{\prime}, t^{\prime}\right)\right\rangle_{M}:=x_{1} x_{1}^{\prime}+x_{2} x_{2}^{\prime}-x_{3} x_{3}^{\prime}+t t^{\prime},
$$

for all $(x, t):=\left(\left(x_{1}, x_{2}, x_{3}\right), t\right)$ and $\left(x^{\prime}, t^{\prime}\right):=\left(\left(x_{1}^{\prime}, x_{2}^{\prime}, x_{3}^{\prime}\right), t^{\prime}\right)$ in $\mathbb{R}^{4}=\mathbb{R}^{3} \times \mathbb{R}$. Indeed, $d h$ determines the family $\left(L_{h+t}(v)\right)_{(v, t) \in \mathbf{H}^{2} \times \mathbb{R}}$ of oriented null lines of $\mathbf{M}^{4}$ defined by:

$$
\forall(v, t) \in \mathbb{H}^{2} \times \mathbb{R}, L_{h+t}(v)=l_{h+t}(v)+\mathbb{R}(v,-1),
$$

where $l_{h+t}(v):=(\nabla h(v),-h(v)-t)$; and this family of null lines determines a 'marginally trapped hedgehog', which is unique up to translations parallel to the time axis, via a 'lightlike co-contact condition':

Theorem 6.(Determination of marginally trapped hedgehogs of $\mathbf{M}^{4}$ by 1 -jet and co-contact condition) For all $t \in \mathbb{R}$, (i) there is a unique map $x: \mathbf{H}^{2} \rightarrow \mathbf{M}^{4}$ of class $C^{\infty}$ satisfying $x(v) \in L_{h+t}(v)$ for all $v \in \mathbf{H}^{2}$ together with the 'lightlike co-contact condition'

$$
\left\langle\delta(d x), v_{M}\right\rangle_{M}=0,
$$

where $\delta$ is the Hodge codifferential on $\mathbf{H}^{2}$ (i.e. the formal adjoint to the exterior differentiation $d$ ) and $v_{M}:=\frac{1}{\sqrt{2}}(v,-1)$, namely

$$
x=x_{l_{h+t}}: \mathbf{H}^{2} \rightarrow \mathbf{M}^{4}, v \mapsto l_{h+t}(v)+\delta(\partial h)(v) v_{M},
$$


where $\partial h(v):=d h / \sqrt{2}$;

(ii) this map $x: \mathbf{H}^{2} \rightarrow \mathbf{M}^{4}$ is such that $x^{*} g=\frac{1}{4}\left(R_{1}-R_{2}\right)^{2} g_{H}$, where $g_{H}$ is the standard metric on $\mathbf{H}^{2}, g$ the first fundamental form on $x\left(\mathbf{H}^{2}\right), x^{*} g$ the pullback of $g$ along $x$ and $R_{1}(v), R_{2}(v)$ the principal radii of curvature of $\mathcal{H}_{h}$ at $x_{h}(v)$;

(iii) for all $v \in \mathbf{H}^{2}, \Delta x(v) \in \mathbb{R} v_{M}$, where $\Delta$ is the Hodge Laplacian on $\mathbf{H}^{2}$, so that the mean curvature vector of $x\left(\mathbf{H}^{2}\right)$ at $x(v)$, say $\vec{H}_{x}(v)$, is parallel to the lightlike vector $\overrightarrow{v_{M}}$ whenever $x_{h}(v)$ is not an umbilical point of $\mathcal{H}_{h}$, that is:

$$
\forall v \in \mathbf{H}^{2} \backslash\left\{v \in \mathbf{H}^{2} \mid R_{1}(v)=R_{2}(v)\right\}, \quad \vec{H}_{x}(v) \in \mathbb{R} \overrightarrow{v_{M}}
$$

Here, the principal radii of curvature of $\mathcal{H}_{h}$ at $x_{h}(v), R_{1}(v)$ and $R_{2}(v)$, are defined as the eigenvalues of $x_{h}: T_{v} \mathbf{H}^{2} \rightarrow T_{x_{h}(v)} \mathcal{H}_{h} \subset T_{v} \mathbf{H}^{2}$.

Definition 3. For all $t \in \mathbb{R}$, we call $\mathcal{H}_{l_{h+t}}:=x_{l_{h+t}}\left(\mathbf{H}^{2}\right)$ the (marginally trapped) hedgehog with support 1-jet $j_{h+t}^{1}: \mathbf{H}^{2} \rightarrow J^{1}\left(\mathbf{H}^{2}\right), v \mapsto(\nabla h(v), h(v)+t)$.

Note that $\sqrt{2} \partial$ stands for the Hodge-Dirac operator $D=d+\delta$ on $\mathbf{H}^{2}$ and the datum of $\partial h:=d h / \sqrt{2}$ is equivalent to the one of the family of 1-jets $\left(j_{h+t}^{1}\right)_{t \in \mathbb{R}}$, where $j_{h+t}^{1}:=(\nabla h, h+t)$. All the marginally trapped hedgehogs of the family $\left(\mathcal{H}_{l_{h+t}}\right)_{t \in \mathbb{R}}$ are equal up to translations parallel to the time axis $\mathbb{R} \overrightarrow{\partial_{t}}$ in $\mathbf{M}^{4}$, where $\overrightarrow{\partial_{t}}:=\left(\overrightarrow{0_{\mathbb{L}^{3}}}, 1\right)$, since: for all $(v, t) \in \mathbf{H}^{2} \times \mathbb{R}, x_{l_{h+t}}(v)=x_{l_{h}}(v)-t \overrightarrow{\partial_{t}}$. Therefore, this family can be regarded as one and only one marginally trapped hedgehog defined up to a translation parallel to the time axis $\mathbb{R} \overrightarrow{\partial_{t}}$ in $\mathbf{M}^{4}$. This hedgehog will be denoted by $\mathcal{H}_{\partial h}$, where $\partial h:=d h / \sqrt{2}$ (the factor $1 / \sqrt{2}$ is chosen for the same reasons as in the case of marginally trapped hedgehogs of $\left.\mathbb{L}^{4}\right)$.

These remarks make natural the following definition:

Definition 4. We call $\mathcal{H}_{\partial h}$ the marginally trapped hedgehog (or, co-hedgehog) of $\mathbf{M}^{4}$ with support differential $\partial h:=d h / \sqrt{2}$. Anyone of the marginally trapped hedgehogs $\mathcal{H}_{l_{h+t}},(t \in \mathbb{R})$, will be regarded as a representative of $\mathcal{H}_{\partial h}$ in $\mathbf{M}^{4}$. We will say that $\mathcal{H}_{\partial h}$ is the co-evolute of $\mathcal{H}_{h}$ in $\mathbf{M}^{4}$.

Thus, once the lightlike 'co-contact condition' $\left\langle\delta(d x), v_{M}\right\rangle_{M}=0$ is fixed, the hedgehog $\mathcal{H}_{\partial h}$ is defined in $\mathbf{M}^{4}$ as the 'co-envelope' of the family of oriented null lines determined by its support differential $\partial h$ in the same way as, once the contact condition $\langle d x, v\rangle_{L}=0$ is fixed, the hedgehog $\mathcal{H}_{h}$ is defined in $\mathbb{L}^{3}$ as the envelope of the family of cooriented spacelike planes determined by its support function $h$. Of course, it is important to note that $\mathcal{H}_{h}$ and $\mathcal{H}_{\partial h}$ are not assumed to be embedded surfaces of respectively $\mathbb{L}^{3}$ and $\mathbf{M}^{4}: \mathcal{H}_{h}$ and $\mathcal{H}_{\partial h}$ may possibly be singular and self-intersecting. 


\subsubsection{Geometrical interpretation of co-hedgehogs of $\mathbb{M}^{4}$}

First, let us remind some basic facts about the curvature of hedgehogs of $\mathbb{L}^{3}$. Since the parametrization $x_{h}: \mathbf{H}^{2} \rightarrow \mathcal{H}_{h} \subset \mathbb{L}^{3}$ can be regarded as the inverse of the Gauss map, the Gauss curvature $K_{h}$ of $\mathcal{H}_{h}$ at $x_{h}(v)$ is given by $K_{h}(v)=$ $1 / \operatorname{det}\left[T_{v} x_{h}\right]$, where $T_{v} x_{h}$ is the tangent map of $x_{h}$ at $v$. Therefore, singularities of $\mathcal{H}_{h}$ are the points at which its Gauss curvature becomes infinite. For all $v \in \mathbf{H}^{2}$, the tangent map of $x_{h}$ at the point $u$ is $T_{u} x_{h}=H_{h}(v)-h(v) I d_{T_{v} \mathbf{H}^{2}}$, where $H_{h}(v)$ is the symmetric endomorphism associated with the Hessian of $h$ at $v$. Consequently, if $\lambda_{1}(v)$ and $\lambda_{2}(v)$ are the eigenvalues of the Hessian of $h$ at $v$ then $R_{1}(v):=\left(\lambda_{1}-h\right)(u)$ and $R_{2}(v):=\left(\lambda_{2}-h\right)(v)$ can be interpreted as the principal radii of curvature of $\mathcal{H}_{h}$ at $x_{h}(v)$, and the so-called curvature function $R_{h}:=1 / K_{h}$ is given by

$$
R_{h}(v)=\operatorname{det}\left[H_{i j}(u)-h(u) \delta_{i j}\right]=\left(R_{1} R_{2}\right)(v),
$$

where $\delta_{i j}$ are the Kronecker symbols and $\left(H_{i j}(v)\right)$ the Hessian of $h$ at $v$ with respect to an orthonormal frame on $\mathbf{H}^{2}$. In computations, it is often more convenient to replace $h$ by its positively 1 -homogeneous extension to the interior of the light cone $\mathcal{U}=\left\{x \in \mathbb{L}^{3} \mid\langle x, x\rangle_{L}<0\right\}$, that is, by

$$
\varphi(x):=\|x\|_{L} h\left(\frac{x}{\|x\|_{L}}\right),
$$

for $x \in \mathcal{U}$, where $\|x\|_{L}=\sqrt{-\langle x, x\rangle_{L}}$. A straightforward computation gives:

(i) $x_{h}$ is the restriction of the Lorentzian gradient $\nabla_{L} \varphi:=\left(\frac{\partial \varphi}{\partial x_{1}}, \frac{\partial \varphi}{\partial x^{2}},-\frac{\partial \varphi}{\partial x_{3}}\right)$ of $\varphi$ to the unit sphere $\mathbf{H}^{2}$;

(ii) For all $v \in \mathbf{H}^{2}$, the tangent map $T_{v} x_{h}$ identifies with the symmetric endomorphism associated with the Hessian of $\varphi$ at $v$.

In order to bring out our geometrical interpretation of the co-evolute $\mathcal{H}_{\partial h}$, we now give another expression for $x_{l_{h}}$. For all $v \in \mathbf{H}^{2}$, we have

$$
\begin{aligned}
x_{l_{h}}(v) \quad & :=l_{h}(v)+\delta(\partial h)(v) v_{M}=(\nabla h(v),-h(v))+\frac{\Delta h(v)}{2}(v,-1) \\
& =\left(\nabla h(v)+\frac{\Delta h(v)}{2} v,-h(v)-\frac{\Delta h(v)}{2}\right) \\
& =\left(x_{h}(v)-R_{(-1, h)}(v) v, R_{(-1, h)}(v)\right)=\left(c_{h}(v), R_{(-1, h)}(v)\right)
\end{aligned}
$$

where $\Delta$ is the Hodge Laplacian on $\mathbf{H}^{2}, c_{h}(v)$ is the midpoint of the segment connecting the two centers of principal curvatures of $\mathcal{H}_{h}$ at $x_{h}(v)$ and, $R_{(-1, h)}:=-\left(h+\frac{\Delta h}{2}\right)=\frac{1}{2}\left(R_{1}+R_{2}\right)$ is the mean radius of principal curvature of $\mathcal{H}_{h}$. Thus, for all $v \in \mathbf{H}^{2}, x_{l_{h}}(v)$ can be interpreted geometrically as the middle pseudosphere (or possibly non-cooriented light cone) of $\mathcal{H}_{h}$ at $x_{h}(v)$ that is cooriented by the vector $v$, which is normal to it, by identifying $\mathbf{M}^{4}$ to the 
space $\Pi$ of cooriented pseudospheres and (non-cooriented) light cones of $\mathbb{L}^{3}$ via the bijection

$$
\begin{array}{cl}
\mathbf{M}^{4}=\mathbb{L}^{3} \times \mathbb{R} & \rightarrow \Pi \\
(a, r)=\left(\left(a_{1}, a_{2}, a_{3}\right), r\right) & \mapsto H^{2}(a ; r),
\end{array}
$$

where $H^{2}(a ; r)$ is the two-sheeted hyperboloid (pseudosphere) with equation $\langle x-a, x-a\rangle_{L}=-r^{2}$ that is cooriented so that its upper sheet $H_{+}^{2}(a ; r):=$ $H^{2}(a ; r) \cap\left\{x_{3}>a_{3}\right\}$ is cooriented by its future (resp. pass) pointing normal if $r>0$ (resp. $r<0$ ) holds, and the (non-cooriented) light cone with apex at $a$ if $r=0$ holds. Thus:

Proposition 2. The co-evolute $\mathcal{H}_{\partial h}$ of $\mathcal{H}_{h}$ can be regarded as the locus of all the middle cooriented pseudospheres (or non-cooriented light cones) of $\mathcal{H}_{h} \subset \mathbb{L}^{3}$ in $\mathbf{M}^{4} \cong \Pi$.

\subsection{Towards other spaces and generalizations}

As already mentioned, most of our results extend, without much change, to higher dimensions. Furthermore, the hedgehog theory is of course not restricted to the only two examples we have considered above. For instance, we could have adapted our presentation to Fuchsian hedgehogs, which were introduced by François Fillastre [4]. We could also have considered 'multihedgehogs' or ' $N$-hedgehogs' as they were introduced by Langevin, Levitt and Rosenberg [6], provided of course that we pay proper attention to the fact that an $N$-hedgehog may have parabolic points (i.e., points where their Gauss curvature vanishes, and thus, points at which their curvature function is not defined) for $N \geq 2$. Recall that an $N$-hedgehog of $\mathbb{R}^{3}$ is any envelope of a family of cooriented planes of $\mathbb{R}^{3}$ such that the number of cooriented support planes with a given coorienting unit normal vector is finite and constant equal to $N$ (at least for an open dense set of directions). Thus 1-hedgehogs of $\mathbb{R}^{3}$ are simply hedgehogs. But in fact, all these geometrical objects of different spaces can be regarded as wavefronts of a special class of Legendrian submanifolds (i.e. images of Legendrian maps) of a given metric contact manifold. Before going on, we first recall some basic definitions and facts on contact, symplectic and almost-hermitian structures, and next present and study hedgehogs and marginally trapped hedgehogs in this setting.

\subsubsection{Basic definitions and facts}

\section{Contact manifolds and metric contact manifolds}

A contact structure on an oriented $(2 n+1)$-dimensional $C^{\infty}$-manifold $M$ is the datum of a smooth field $V$ of tangent hyperplanes on $M$, called contact hyperplanes, satisfying the following condition of maximal non-integrability: any (and hence every) 1 -form $\alpha$ defining $V$ (i.e. such that $V=\operatorname{Ker}(\alpha)$ ) satisfies $\alpha \wedge(d \alpha)^{n} \neq 0$ everywhere on $M$. Any 1 -form $\alpha$ defining such a maximally 
non-integrable hyperplane field $V$ on $M$ is called a contact form on $M$. Given such a contact structure (or a contact form $\alpha$ defining it), the pair $(M, V)$ (or the pair $(M, \alpha)$ if we want to fix the contact form defining $V)$ is then called a contact manifold. On $(M, \alpha)$, the Reeb vector field $\xi_{\alpha}$ associated to the contact form $\alpha$ is defined to be the unique smooth vector field satisfying

$$
\alpha\left(\xi_{\alpha}\right)=1 \quad \text { and } \quad \xi_{\alpha} \in \operatorname{Ker}(d \alpha) .
$$

A submanifold $L$ of a contact manifold $(M, V)$ is said to be integral if $T_{m} L \subset V_{m}$ for all $m \in L$. A Legendrian submanifold of $(M, V)$ is an integral submanifold of $(M, V)$ with maximal dimension $n=(\operatorname{dim} M-1) / 2$. A fibration of a contact manifold is said to be Legendrian if all its fibers are Legendrian submanifolds.

Let $i: L \rightarrow E$ be an immersed Legendrian submanifold $L$ in the total space of a Legendrian fibration $\pi: E \rightarrow B$. The restriction of $\pi$ to $L$, that is $x=\pi \circ i: L \rightarrow B$ is called a Legendrian map and its image $x(L)$ in $B$ is called its Legendrian front or wavefront.

Example. Unit tangent bundles of Riemannian manifolds are among the most classical examples of contact manifolds. Let us recall briefly how this is done. Let $(M, g)$ be a Riemannian manifold and let

$$
U T M=\{u \in T M \mid g(u, u)=1\}
$$

be its unit tangent bundle with canonical projection $\pi: U T M \rightarrow M$; the metric $g$ induces a contact form $\alpha$ (and thus a contact structure $V$ ) on $U T M$ as follows: for any $u \in U T M$ and $v \in T_{u}(U T M)$, we let

$$
\alpha_{u}(v)=g\left(u, T_{u} \pi(v)\right),
$$

where $T_{u} \pi(v)=\pi_{*}(v)$ is the pushforward along $\pi$ of the vector $v$. Moreover, $\pi: U T M \rightarrow M$ is an example of a Legendrian fibration.

In particular, if we let

$$
\alpha_{(x, u)}:=\langle u, d x\rangle=\sum_{i=0}^{n+1} u_{i} d x_{i}
$$

for all $(x, u) \in U \mathbb{R}^{n+1}=\mathbb{R}^{n+1} \times \mathbb{S}^{n}$, where $\left(x_{1}, \cdots, x_{n+1} ; u_{1}, \cdots, u_{n+1}\right)$ are the canonical coordinate functions on $U \mathbb{R}^{n+1}=\mathbb{R}^{n+1} \times \mathbb{S}^{n} \subset \mathbb{R}^{2 n+2}$, we obtain a contact manifold $\left(U \mathbb{R}^{n+1} ; \alpha\right)$.

A contactomorphism from a contact manifold $\left(M_{1}, V_{1}\right)$ to a contact manifold $\left(M_{2}, V_{2}\right)$ is a diffeomorphism $f: M_{1} \rightarrow M_{2}$ that preserves the contact structure, i.e. such that $T f\left(V_{1}\right)=V_{2}$, where $T f: T M_{1} \rightarrow T M_{2}$ denotes the tangent map of $f$. If $V_{i}=\operatorname{Ker}\left(\alpha_{1}\right),(i=1,2)$, this is equivalent to the existence of a nowhere zero function $\lambda: M_{1} \rightarrow M_{2}$ such that $f^{*} \alpha_{2}=\lambda \alpha_{1}$.

Example. Another example of a contact manifold is defined as follows: on the manifold $T \mathbb{S}^{n} \times \mathbb{R}$, where the tangent bundle $T \mathbb{S}^{n}$ is identified with

$$
\left\{(u, p) \in\left(\mathbb{R}^{n+1}\right)^{2} \mid\|u\|=1 \text { and }\langle u, p\rangle=0\right\}
$$


$(\|$.$\| and \langle.,$.$\rangle denoting respectively the Euclidean norm and scalar product in$ $\left.\mathbb{R}^{n+1}\right)$, we define a contact form $\beta$ by putting $\beta_{(u, p, z)}:=d z-p d u$ for all $(u, p, z) \in T \mathbb{S}^{n} \times \mathbb{R}$. Moreover

$$
\begin{aligned}
f: \quad U \mathbb{R}^{n+1} & =\mathbb{R}^{n+1} \times \mathbb{S}^{n} \rightarrow T \mathbb{S}^{n} \times \mathbb{R} \\
(x, u) & \mapsto(u, x-\langle x, u\rangle u,\langle x, u\rangle)
\end{aligned}
$$

is a diffeomorphism such that $f^{*} \beta=\alpha$, and hence a contactomorphism from $\left(U \mathbb{R}^{n+1}, \alpha\right)$ to $\left(T \mathbb{S}^{n} \times \mathbb{R}, \beta\right)$.

A metric contact manifold is defined to be a tuple $(M, g, \alpha, J)$, where $(M, g)$ is a Riemannian manifold, $\alpha$ a smooth 1 -form on $M$ and $J$ a section of the endomorphism bundle End $(T M)$ which satisfy the three following conditions:

(i) $\alpha\left(\xi_{\alpha}\right)=1$, where $\xi_{\alpha}$ is the metric dual of $\alpha$;

(ii) $d \alpha(X, Y)=g(J X, Y)$ for any vector fields $X, Y$ on $M$;

(iii) $J^{2} X=-X+\alpha(X) \xi_{\alpha}$ for any vector field $X$ on $M$.

Then $(M, \operatorname{Ker}(\alpha))$ is a contact manifold (i.e. $\alpha \wedge(d \alpha)^{n} \neq 0$ on $\left.M\right), \xi_{\alpha}$ is the Reeb vector field associated to $\alpha, J \xi_{\alpha}=0$ and $g$ is determined by $\alpha$ and $J$ through the equality $g(X, Y)=\alpha(X) \alpha(Y)+d \alpha(X, J Y)$, (see e.g. [14]).

Example. In the case of hedgehogs of $\mathbb{R}^{n+1}$, we will consider the metric contact manifold $\left(U \mathbb{R}^{n+1}, g, \alpha, J\right)$, where $g$ is the Riemannian product metric on $U \mathbb{R}^{n+1}=\mathbb{R}^{n+1} \times \mathbb{S}^{n}$ and $J: T U \mathbb{R}^{n+1} \rightarrow T U \mathbb{R}^{n+1},(X, Q) \mapsto(Q,\langle X, q\rangle q-X)$.

\section{Hedgehogs as Legendrian fronts}

Let us consider first the case where $(M, g)=\left(\mathbb{R}^{n+1}, g_{\text {can }}\right)$, where $g_{\text {can }}=$ $\langle.,$.$\rangle is the canonical Euclidean metric. Let \mathcal{H}_{h}$ be a hedgehog of $\mathbb{R}^{n+1}$ with support function $h \in C^{\infty}\left(\mathbb{S}^{n} ; \mathbb{R}\right)$. Let us recall that its natural parametrization $x_{h}: \mathbb{S}^{n} \rightarrow \mathbb{R}^{n+1}, u \mapsto x_{h}(u)=h(u) u+(\nabla h)(u)$ can be interpreted as the inverse of its Gauss map. Thus it appears that

$$
\begin{aligned}
i_{h}: & \mathbb{S}^{n} \rightarrow U \mathbb{R}^{n+1}=\mathbb{R}^{n+1} \times \mathbb{S}^{n} \\
& u \mapsto\left(x_{h}(u), u\right)
\end{aligned}
$$

is the immersion of a Legendrian submanifold in $U \mathbb{R}^{n+1}$ of which $\mathcal{H}_{h}$ is the Legendrian front in $\mathbb{R}^{n+1}$ and $x_{h}=\pi \circ i_{h}$ the corresponding Legendrian map. Recall that on $U \mathbb{R}^{n+1}$, the contact form and the associated Reeb vector field are respectively given by

$$
\alpha_{(x, u)}:=\langle u, d x\rangle=\sum_{i=0}^{n+1} u_{i} d x_{i} \quad \text { and } \quad \xi(x, u):=\left(u ; 0_{T_{u} \mathbb{S}^{n}}\right),
$$

for all $(x, u) \in U \mathbb{R}^{n+1}$, where $\left(x_{1}, \cdots, x_{n+1} ; u_{1}, \cdots, u_{n+1}\right)$ are the canonical coordinate functions on $U \mathbb{R}^{n+1}=\mathbb{R}^{n+1} \times \mathbb{S}^{n} \subset \mathbb{R}^{2 n+2}$.

Thus, hedgehogs of $\mathbb{R}^{n+1}$ are the Legendrian fronts of those Legendrian submanifolds of $\left(U \mathbb{R}^{n+1}, \alpha\right)$ whose Legendrian maps can be interpreted as the inverse of the Gauss map of their image (i.e. of the Legendrian front). 


$$
\begin{array}{ccc}
\mathbb{S}^{n} \stackrel{i_{h}}{\rightarrow} & i_{h}\left(\mathbb{S}^{n}\right) \subset\left(U \mathbb{R}^{n+1}, \operatorname{Ker}(\alpha)\right) \\
x_{h} \searrow & \downarrow \pi \\
& \\
& \mathcal{H}_{h} \subset \mathbb{R}^{n+1} .
\end{array}
$$

This can of course be adapted to hedgehogs of other spaces. We can, for instance, replace:

$\cdot\left(\mathbb{R}^{n+1},\langle.,\rangle.\right)$ by the Lorentzian-Minkowski $(n+1)$-space $\mathbb{L}^{n+1}=\left(\mathbb{R}^{n+1},\langle., .\rangle_{L}\right)$, where

$$
\left\langle x, x^{\prime}\right\rangle_{L}=\sum_{k=0}^{n} x_{k} x_{k}^{\prime}-t t^{\prime}
$$

for all $x:=\left(\left(x_{1}, \ldots, x_{n}\right), t\right)$ and $x:=\left(\left(x_{1}^{\prime}, \ldots, x_{n}^{\prime}\right), t^{\prime}\right)$ in $\mathbb{L}^{n+1}=\mathbb{R}^{n} \times \mathbb{R}$;

- $\mathbb{S}^{n}$ by $\mathbf{H}^{n}:=\left\{x=\left(\left(x_{1}, \ldots, x_{n}\right), t\right) \in \mathbb{L}^{n+1} \mid\langle x, x\rangle_{L}=-1\right\}$;

. $U \mathbb{R}^{n+1}=\mathbb{R}^{n+1} \times \mathbb{S}^{n}$ by $T_{-1} \mathbf{H}^{n}:=\mathbb{L}^{n+1} \times \mathbf{H}^{n}$;

$\cdot x_{h}: \mathbb{S}^{n} \rightarrow \mathbb{R}$ by $x_{h}: \mathbf{H}^{n} \rightarrow \mathbb{L}^{n+1}, v \mapsto x_{h}(v)=(\nabla h)(v)-h(v) v ;$

$\cdot i_{h}: \mathbb{S}^{n} \rightarrow U \mathbb{R}^{n+1}$ by $i_{h}: \mathbf{H}^{n} \rightarrow T_{-1} \mathbf{H}^{n}, v \mapsto\left(x_{h}(v), v\right)$;

- $\alpha_{(x, u)}$ by $\alpha_{(x, v)}:=\langle v, d x\rangle_{L}$ and $\xi_{\alpha}(x, u)$ by $\xi_{\alpha}(x, v):=\left(v ; 0_{T_{v} \mathbf{H}^{n}}\right)$.

\section{Symplectic and almost-hermitian structures}

A symplectic structure on a $2 n$-dimensional $C^{\infty}$-manifold $M$ is a closed differentiable 2-form $\omega$ that is nondegenerate (i.e. such that: $\omega(u, v)=0$ for all $u \in T_{m} M$ implies $\left.v=0_{T_{m} M}\right)$. The pair $(M, \omega)$ is then called a symplectic manifold.

A submanifold $L$ of a symplectic manifold $(M, \omega)$ is said to be Lagrangian if the restriction of $\omega$ to $L$ is equal to 0 and $\operatorname{dim} L=(\operatorname{dim} M) / 2$. A fibration $\pi: E \rightarrow B$ of a symplectic manifold $E$ is called a Lagrangian fibration if all the fibers are Lagrangian submanifolds. Let $i: L \rightarrow E$ be an immersed Lagrangian submanifold $L$ in the total space of a Lagrangian fibration $\pi: E \rightarrow B$. The restriction of $\pi$ to $L$, that is $x=\pi \circ i: L \rightarrow B$ is called a Lagrangian map.

Given any contact manifold $(M, \alpha)$, there is a canonical symplectic structure on $M \times \mathbb{R}$, which is given by $\omega=d\left(e^{t} \alpha\right)$. We say that $\left(M \times \mathbb{R}, d\left(e^{t} \alpha\right)\right)$ is the symplectization of the contact manifold $(M, \alpha)$.

Example. The symplectization of the contact manifold $\left(U \mathbb{R}^{3}, \alpha\right)$, where $\alpha_{(y, u)}:=\langle u, d y\rangle$ for all $(y, u) \in \mathbb{R}^{3} \times \mathbb{S}^{2}$, can be regarded as the symplectic manifold $\left(\mathbb{L}^{4} \times \mathbb{S}^{2}, \omega\right)$, where $\omega_{(y, t, u)}=d\left(e^{t} \alpha_{(y, u)}\right)$, and $\pi_{L}: \mathbb{L}^{4} \times \mathbb{S}^{2} \rightarrow \mathbb{R}^{3}$, $(y, t, u) \mapsto y$ is a Lagrangian fibration. Moreover, for any $h \in C^{\infty}\left(\mathbb{S}^{2} ; \mathbb{R}\right)$,

$$
\begin{gathered}
I_{h}: \quad \mathbb{S}^{2} \times \mathbb{R} \rightarrow \mathbb{L}^{4} \times \mathbb{S}^{2} \\
(u, t) \mapsto\left(x_{h-t}(u), t, u\right)
\end{gathered}
$$


appears to be the immersion of a Lagrangian submanifold $\mathcal{L}_{h}:=I_{h}\left(\mathbb{S}^{2} \times \mathbb{R}\right)$ in $\mathbb{L}^{4} \times \mathbb{S}^{2}$. Note that this Lagrangian submanifold $\mathcal{L}_{h}$ of $\mathbb{L}^{4} \times \mathbb{S}^{2}$ is obtained by lifting to $\mathbb{L}^{4} \times \mathbb{S}^{2}$ the family $\left(i_{h-t}\right)_{t \in \mathbb{R}}$ of Legendrian immersions $i_{h-t}: \mathbb{S}^{2} \rightarrow U \mathbb{R}^{3}$, $u \mapsto\left(x_{h-t}(u), u\right)$ whose Legendrian fronts $x_{h-t}\left(\mathbb{S}^{2}\right)$ form the family of parallel hedgehogs $\left(\mathcal{H}_{h-t}\right)_{t \in \mathbb{R}}$ in $\mathbb{R}^{3}$.

A symplectomorphism from a symplectic manifold $\left(M_{1}, \omega_{1}\right)$ to a symplectic manifold $\left(M_{2}, \omega_{2}\right)$ is a diffeomorphism $f: M_{1} \rightarrow M_{2}$ that preserves the symplectic structure, i.e. such that $f^{*} \omega_{2}=\omega_{1}$.

Example. It is easy to check that

$$
\begin{array}{cl}
f: \quad & \left(\mathbb{L}^{4} \times \mathbb{S}^{2}, d\left(e^{t} \alpha\right)\right) \rightarrow\left(T\left(\mathbb{S}^{2}\right) \times \mathbb{R}^{2}, d\left(e^{t} \beta\right)\right) \\
& (y, t, u) \mapsto(u, y-\langle y, u\rangle u,\langle y, u\rangle, t)
\end{array}
$$

is a symplectomorphism between the symplectizations of $\left(U \mathbb{R}^{3}, \alpha\right)$ and $\left(T\left(\mathbb{S}^{2}\right) \times \mathbb{R}, \beta\right)$.

An almost complex manifold is defined to be a tuple $(M, J)$, where $(M, g)$ is a Riemannian manifold and $J$ an almost-complex structure on $T M$, that is, a vector bundle endomorphism $J: T M \rightarrow T M$ such that $J^{2}=-1$. An almost Hermitian manifold is defined to be a tuple $(M, g, J)$, where $(M, J)$ is an almost complex manifold and $g$ an almost-hermitian metric on $(M, J)$, that is, a Riemannian metric on $M$ satisfying:

$$
\forall m \in M, \forall(X, Y) \in\left(T_{m} M\right)^{2}, \quad g(X, J Y)=-g(J X, Y) .
$$

To any metric contact manifold $(M, g, \alpha, J)$, we can also associate a manifold $\widehat{M}=M \times \mathbb{R}$ which carries an almost-hermitian structure $(\widehat{g}, \widehat{J})$ extending the one we have on the contact distribution $V=\operatorname{Ker}(\alpha)$ : indeed, $(\widehat{C}, \widehat{g}, \widehat{J})$ is almost Hermitian if we let $\widehat{g}=g+d t^{2}, \widehat{J}_{\mid V}=J_{\mid V}, \widehat{J} \xi_{\alpha}=-\partial_{t}$ and $\widehat{J} \partial_{t}=\xi_{\alpha}$, where $t$ is the coordinate on the $\mathbb{R}$ factor (see e.g. [14]).

\subsubsection{Marginally trapped hedgehogs of an associated Lagrangian submanifold}

Given any $h \in C^{\infty}\left(\mathbb{S}^{2} ; \mathbb{R}\right)$, let us consider the marginally trapped hedgehog $\mathcal{H}_{\partial h}$ of $\mathbb{L}^{4}$ with support differential $\partial h:=d h / \sqrt{2}$. As we have seen above, $\left(\mathbb{L}^{4} \times \mathbb{S}^{2}, \omega:=d\left(e^{t} \alpha\right)\right)$ can be regarded as the symplectization of the contact manifold $\left(U \mathbb{R}^{3}, \alpha\right)$, where $\alpha:=\langle u, d y\rangle, \pi_{L}: \mathbb{L}^{4} \times \mathbb{S}^{2} \rightarrow \mathbb{R}^{3},(y, t, u) \mapsto y$ is a Lagrangian fibration and $I_{h}: \mathbb{S}^{2} \times \mathbb{R} \rightarrow \mathbb{L}^{4} \times \mathbb{S}^{2},(u, t) \mapsto\left(x_{h-t}(u), t, u\right)$ is the immersion of a Lagrangian submanifold $\mathcal{L}_{h}:=I_{h}\left(\mathbb{S}^{2} \times \mathbb{R}\right)$ in $\mathbb{L}^{4} \times \mathbb{S}^{2}$. If we endow the symplectic manifold $\left(\mathbb{L}^{4} \times \mathbb{S}^{2}, \omega\right)$ with the Lorentzian metric $g_{L}:=g-d t^{2}$, where $g$ is the Riemannian product metric on $U \mathbb{R}^{3}=\mathbb{R}^{3} \times \mathbb{S}^{2}$, then

$$
\begin{aligned}
i_{\partial h}: & \mathbb{S}^{2} \rightarrow\left(\mathbb{L}^{4} \times \mathbb{S}^{2}, g_{L}\right) \\
& u \mapsto\left(x_{\partial h}(u), u\right)=\left(\nabla h(u)+\partial^{2} h(u) u, h(u)-\partial^{2} h(u), u\right)
\end{aligned}
$$


is the parametrization of a marginally trapped surface $\mathcal{I}_{\partial h}:=i_{\partial h}\left(\mathbb{S}^{2}\right)$ included in the Lagrangian submanifold $\mathcal{L}_{h}$ of $\mathbb{L}^{4} \times \mathbb{S}^{2}$ : indeed, $\mathcal{I}_{\partial h}$ is spacelike (i.e. its induced metric is Riemannian) and its mean curvature vector $\mathcal{H}_{i_{\partial h}}$ is lightlike at each point. More precisely, it is a routine to check that:

(i) $i_{\partial h}^{*} g=\left(1+\frac{1}{4}\left(R_{1}-R_{2}\right)^{2}\right) g_{\mathbb{S}}$, where $g_{S}$ is the standard metric on $\mathbb{S}^{2}$, $g$ the first fundamental form on $\mathcal{I}_{\partial h}, i_{\partial h}^{*} g$ the pullback of $g$ along $i_{\partial h}$ and $R_{1}(u), R_{2}(u)$ the principal radii of curvature of $\mathcal{H}_{h}$ at $x_{h}(u)$;

(ii) for all $u \in \mathbb{S}^{2}, \mathcal{H}_{i_{\partial h}}\left(i_{\partial h}(u)\right) \in \mathbb{R}\left(\xi_{\alpha}-\partial_{t}\right)$, where $\xi_{\alpha}:=\left(u, 0,0_{T_{u} \mathbb{S}^{2}}\right)$, $\partial_{t}:=\left(0,1,0_{T_{u} \mathbb{S}^{2}}\right)$ and hence $\xi_{\alpha}-\partial_{t}:=\left(u,-1,0_{T_{u} \mathbb{S}^{2}}\right) \in T_{i_{\partial h}(u)} \mathcal{I}_{\partial h} \subset \mathbb{L}^{4} \times T_{u} \mathbb{S}^{2}$. Thus, $\mathcal{H}_{\partial h}$ is the image of $\mathcal{I}_{\partial h}$ under the projection $\pi_{L}: \mathbb{L}^{4} \times \mathbb{S}^{2} \rightarrow \mathbb{L}^{4}$, and we have the following commutative diagram:

$$
\begin{array}{ccc}
\mathbb{S}^{2} \stackrel{i_{\partial h}}{\rightarrow} & \mathcal{I}_{\partial h} \text { marginally trapped in } \mathcal{L}_{h} \subset\left(\mathbb{L}^{4} \times \mathbb{S}^{2}, g_{L}\right) \\
x_{\partial h} \searrow & \downarrow \pi_{L} \\
& & \mathcal{H}_{\partial h} \text { marginally trapped in } \mathbb{L}^{4} .
\end{array}
$$

In other words, any marginally trapped hedgehog $x_{\partial h}: \mathbb{S}^{2} \rightarrow \mathcal{H}_{\partial h} \subset \mathbb{L}^{4}$ lifts to a marginally trapped surface $\mathcal{I}_{\partial h}$ of the Lagrangian submanifold $\mathcal{L}_{h}$ of $\left(\mathbb{L}^{4} \times \mathbb{S}^{2}, \omega, g_{L}\right)$ that is deduced from the family of parallel hedgehogs $\left(\mathcal{H}_{h-t}\right)_{t \in \mathbb{R}}$.

Now, if $M$ is another 6-dimensional $C^{\infty}$-manifold endowed with a symplectic structure $\omega_{M}$ and a Lorentzian metric $g_{M}$, and if $f: \mathbb{L}^{4} \times \mathbb{S}^{2} \rightarrow M$ is a diffeomorphism preserving both the symplectic structure and the Lorentzian metric, then $f\left(\mathcal{I}_{\partial h}\right)$ is a marginally trapped surface of the Lagrangian submanifold $f\left(\mathcal{L}_{h}\right)$ of $\left(M, \omega_{M}, g_{M}\right)$. For instance, if we use the diffeomorphism

$$
\begin{array}{cc}
f: \quad & \mathbb{L}^{4} \times \mathbb{S}^{2} \rightarrow T\left(\mathbb{S}^{2}\right) \times \mathbb{R}^{2} \\
& (y, t, u) \mapsto(u, y-\langle y, u\rangle u,\langle y, u\rangle, t),
\end{array}
$$

to transport the Lorentzian metric $g_{L}$ to a Lorentzian metric $g_{M}$ on $T\left(\mathbb{S}^{2}\right) \times \mathbb{R}^{2}$, then we see that

$$
\begin{aligned}
j_{\partial h}: \quad \mathbb{S}^{2} & \rightarrow\left(T\left(\mathbb{S}^{2}\right) \times \mathbb{R}^{2}, d\left(e^{t} \beta\right), g_{M}\right) \\
u & \mapsto\left(u, \nabla h(u), \partial^{2} h(u), h(u)-\partial^{2} h(u)\right)
\end{aligned}
$$

defines a marginally trapped surface $j_{\partial h}\left(\mathbb{S}^{2}\right)$ of the Lagrangian submanifold of $\left(T\left(\mathbb{S}^{2}\right) \times \mathbb{R}^{2}, d\left(e^{t} \beta\right)\right)$ that is parametrized by:

$$
\begin{aligned}
J_{h}: \quad & \mathbb{S}^{2} \times \mathbb{R} \rightarrow T\left(\mathbb{S}^{2}\right) \times \mathbb{R}^{2} \\
& (u, t) \mapsto\left(j_{h-t}^{1}(u), t\right):=(u, \nabla h(u), h(u)-t, t) .
\end{aligned}
$$

Let us conclude this section with a remark concerning the definition of the marginally trapped hedgehog $x_{\partial h}: \mathbb{S}^{2} \rightarrow \mathcal{H}_{\partial h} \subset \mathbb{L}^{4}$, where $\mathbb{L}^{4}$ is identified 
with $\Sigma$. For any $u \in \mathbb{S}^{2}, x=x_{\partial h}(u)$ is the (possibly reduced to a point) cooriented sphere of $\mathbb{R}^{3}$ that satisfies the following system of conditions:

$$
\left\{\begin{array}{l}
(1) x \in L_{h}(u) \\
(2)\left\langle x, \Delta u_{L}\right\rangle=\delta(d h)(u),
\end{array}\right.
$$

(see Section 1). The first condition simply ensures that the 'contact element' $i_{h}(u):=\left(x_{h}(u), u\right)$ is in oriented contact with the sphere $x_{\partial h}(u)$ (i.e. $x_{h}(u)$ belongs to the sphere $x_{\partial h}(u)$ and $u$ is the unit normal to $x_{\partial h}(u)$ at $\left.x_{h}(u)\right)$. The second condition (the co-contact one) then ensures that $x_{\partial h}(u)$ is more precisely the oriented middle sphere (or unoriented middle point) $S\left(c_{h}(u) ; R_{(1, h)}(u)\right)$.

\section{Proof of the main results}

We first prove Theorem 1 and Theorem 6, which is its analogue in $\mathbf{M}^{4}$.

Proof of Theorem 1. In this proof, $\nabla_{S}$ (resp. $\nabla$ ) stands for the gradient on $\mathbb{S}^{2}$ (resp. $\mathbb{R}^{3}$ ), $\Delta_{S}$ (resp. $\Delta$ ) for the Laplace-Beltrami operator on $\mathbb{S}^{2}$ (resp. $\mathbb{R}^{3}$ ) and the Laplace-Beltrami operator of a vector function $X: \mathbb{S}^{2} \rightarrow \mathbb{L}^{4}$, $u \mapsto\left(X_{i}(u)\right)_{i=1}^{4}$ is understood to be the vector function $\Delta_{S} X: \mathbb{S}^{2} \rightarrow \mathbb{L}^{4}$, $u \mapsto\left(\Delta_{S} X_{i}(u)\right)_{i=1}^{4}$, where $\Delta_{S} X_{i}$ is the Laplace-Beltrami operator of the coordinate function $X_{i}$ in the intrinsic metric on $\mathbb{S}^{2},(1 \leq i \leq 4)$.

(i) For all $t \in \mathbb{R}$, let $x: \mathbb{S}^{2} \rightarrow \mathbb{L}^{4}$ be any $C^{\infty}$ map such that

$$
x(u) \in L_{h+t}(u):=\left\{l_{h+t}(u)\right\}+\mathbb{R} u_{L}
$$

for all $u \in \mathbb{S}^{2}$. Then there exists some $\lambda \in C^{\infty}\left(\mathbb{S}^{2} ; \mathbb{R}\right)$ such that $x$ is of the form

$$
\begin{aligned}
X_{\lambda}: & \mathbb{S}^{2} \rightarrow \mathbb{L}^{4} \\
& u \mapsto\left(\nabla_{S} h(u), h(u)-t\right)+\lambda(u)(u,-1)=\left(x_{h}(u)+f(u) u, t-f(u)\right),
\end{aligned}
$$

where $f:=\lambda-h$. For all $u \in \mathbb{S}^{2}$, we have:

$$
\Delta_{S} X_{\lambda}(u)=\left(\Delta_{S} x_{h}(u)+\Delta_{S}(f(u) u),-\left(\Delta_{S} f\right)(u)\right) .
$$

Now, $\Delta_{S} x_{h}=\Delta_{S}(\nabla \varphi)=\Delta(\nabla \varphi)=\nabla(\Delta \varphi)$, where $\varphi$ is the positively 1homogeneous extension of $h$ to $\mathbb{R}^{3} \backslash\{0\}$, that is,

$$
\varphi(x):=\|x\| h\left(\frac{x}{\|x\|}\right),
$$

for $x \in \mathbb{R}^{3} \backslash\{0\}$, where $\|$.$\| is the Euclidean norm on \mathbb{R}^{3}$. Indeed, $\nabla \varphi$ is positively 0 -homogeneous on $\mathbb{R}^{3} \backslash\{0\}$ and equal to $x_{h}$ on $\mathbb{S}^{2}$. Thus

$$
\left\langle\Delta_{S} x_{h}(u), u\right\rangle=-(\Delta \varphi)(u)=-\left(\Delta_{S} h+2 h\right)(u),
$$


for $u \in \mathbb{S}^{2}$, since $\Delta \varphi$ is positively -1 -homogeneous on $\mathbb{R}^{3} \backslash\{0\}$. Besides, we have:

$$
\begin{aligned}
\Delta_{S}(f(u) u) & =\left(\Delta_{S} f\right)(u) u+2\left(\left\langle\left(\nabla_{S} f\right)(u),\left(\nabla_{S} x_{i}\right)(u)\right\rangle\right)_{i=1}^{3}+f(u)\left(\Delta_{S} i d_{\mathbb{S}^{2}}\right)(u) \\
& =\left(\Delta_{S} f-2 f\right)(u) u+2\left(\nabla_{S} f\right)(u),
\end{aligned}
$$

for all $u \in \mathbb{S}^{2}$. Therefore, we have:

$$
\begin{aligned}
\left\langle\Delta_{S} X_{\lambda}(u), u_{L}\right\rangle_{L}=0 & \Leftrightarrow-\left(\Delta_{S} h+2 h\right)(u)+\left(\Delta_{S} f-2 f\right)(u)-\left(\Delta_{S} f\right)(u)=0 \\
& \Leftrightarrow-\left(\Delta_{S} h+2 h\right)(u)+2(h-\lambda)(u)=0 \\
& \Leftrightarrow \lambda=-\frac{1}{2}\left(\Delta_{S} h\right)(u),
\end{aligned}
$$

for all $u \in \mathbb{S}^{2}$. Thus

$$
x(u)=X_{\lambda}(u)=l_{h+t}(u)-\frac{\left(\Delta_{S} h\right)(u)}{2}(u,-1)=l_{h+t}(u)+\delta(\partial h)(u) u_{L},
$$

for all $u \in \mathbb{S}^{2}$.

(ii) We know that: $x(u)=\left(x_{h}(u), t\right)-R_{(1, h)}(u)(u,-1)$ for all $u \in \mathbb{S}^{2}$, where $R_{(1, h)}:=h-\frac{\Delta h}{2}=\frac{1}{2}\left(R_{1}+R_{2}\right)$ is the mean radius of principal curvature of $\mathcal{H}_{h}$. From this we deduce that

$$
\left(T_{u} x\right)(v)=\left(\left(T_{u} x_{h}\right)(v), 0\right)-\left(d R_{(1, h)}\right)_{u}(v)(u,-1)-R_{(1, h)}(u)(v, 0) ;
$$

and thus that

$$
\left\langle\left(T_{u} x\right)(v),\left(T_{u} x\right)(v)\right\rangle_{L}=\left\langle\left(T_{u} x_{h}\right)(v)-R_{(1, h)}(u) v,\left(T_{u} x_{h}\right)(v)-R_{(1, h)}(u) v\right\rangle,
$$

for all $v \in T_{u} \mathbb{S}^{2}$. Now, considering an orthonormal basis $\left(e_{1}, e_{2}\right)$ of $T_{u} \mathbb{S}^{2}$ made of eigenvectors of $T_{u} x_{h}$ (i.e., $\left(T_{u} x_{h}\right)\left(e_{i}\right)=R_{i}(u) e_{i}$ for $\left.i \in\{1,2\}\right)$, we conclude that: $\forall v \in T_{u} \mathbb{S}^{2}$,

$$
\left(x^{*} g\right)_{u}(v, v):=\left\langle\left(T_{u} x\right)(v),\left(T_{u} x\right)(v)\right\rangle_{L}=\frac{1}{4}\left(R_{1}-R_{2}\right)(u)^{2} g_{S}(v, v) .
$$

(iii) We know that: $\forall u \in \mathbb{S}^{2}, x(u)=\left(x_{h}(u), t\right)-f(u)(u,-1)$, where $f=R_{(1, h)}$. From this, we deduce that: $\forall u \in \mathbb{S}^{2}$,

$$
\left(\Delta_{S} x\right)(u)=\left(\Delta_{S} x_{h}(u)-\Delta_{S}(f(u) u),\left(\Delta_{S} f\right)(u)\right) .
$$

Now: $\forall u \in \mathbb{S}^{2}, \Delta_{S}(f(u) u)=\left(\Delta_{S} f-2 f\right)(u) u+2\left(\nabla_{S} f\right)(u)$, (see above the proof of $(i))$. Besides, $\Delta_{S} x_{h}=\Delta_{S}(\nabla \varphi)=\Delta(\nabla \varphi)=\nabla(\Delta \varphi)$, where $\varphi$ is the positively 1-homogeneous extension of $h$ to $\mathbb{R}^{3} \backslash\{0\}$ (see ib.), so that $\Delta_{S} x_{h}(u)=$ $\nabla_{S}(\Delta \varphi)(u)-(\Delta \varphi)(u) u=2\left(\nabla_{S} f(u)-f(u) u\right)$ for all $u \in \mathbb{S}^{2}$, since $\Delta \varphi$ is 
positively -1-homogeneous on $\mathbb{R}^{3} \backslash\{0\}$, and equal to $2 f$ on $\mathbb{S}^{2}$. Therefore: $\forall u \in \mathbb{S}^{2}$

$$
\left(\Delta_{S} x\right)(u)=-\left(\Delta_{S} f\right)(u)(u,-1)=-\left(\Delta_{S} R_{(1, h)}\right)(u)(u,-1) \in \mathbb{R} u_{L} .
$$

We know that under a conformal change of metric $\widetilde{g}=e^{2 \phi} g$ on a surface $M^{2}$, $\left(\psi \in C^{\infty}\left(M^{2} ; \mathbb{R}\right)\right)$, the Laplace-Beltrami operator $\Delta_{g}$ transforms according to the formula

$$
\Delta_{g}^{\sim} f=e^{-2 \phi} \Delta_{g} f \quad \text { for all } f \in C^{\infty}\left(M^{2} ; \mathbb{R}\right) .
$$

Above, we have demonstrated that $x^{*} g=\frac{1}{4}\left(R_{1}-R_{2}\right)(u)^{2} g_{S}=\left(R_{(1, h)}^{2}-R_{h}\right) g_{S}$, where $R_{h}:=R_{1} R_{2}$ is the curvature function of $\mathcal{H}_{h}$, and that $\left(\Delta_{S} x\right)(u)=$ $-\left(\Delta_{S} R_{(1, h)}\right)(u)(u,-1)$ for all $u \in \mathbb{S}^{2}$. Therefore

$$
\left(\Delta_{x^{*} g} x\right)(u)=-\frac{\Delta_{S} R_{(1, h)}}{R_{(1, h)}^{2}-R_{h}}(u)(u,-1)
$$

outside umbilical points of $\mathcal{H}_{h}$ (i.e., for all $u \in \mathbb{S}^{2}$ such that $R_{1}(u) \neq R_{2}(u)$ ). We also know that the mean vector field of any surface $X: \Omega \subset \mathbb{S}^{2} \rightarrow \mathbb{L}^{4}$, $u \mapsto\left(X_{i}(u)\right)_{i=1}^{4}$ is given by $\vec{H}_{X}=\frac{1}{2}(\Delta X)=\frac{1}{2}\left(\Delta X_{i}\right)_{i=1}^{4}$, where $\Delta X_{i}$ is the Laplace-Beltrami operator of the coordinate function in the intrinsic metric of the surface. Therefore: $\forall u \in \mathbb{S}^{2} \backslash\left\{u \in \mathbb{S}^{2} \mid R_{1}(u)=R_{2}(u)\right\}$,

$$
\vec{H}_{x}(u)=-\frac{\Delta_{S} R_{(1, h)}}{2\left(R_{(1, h)}^{2}-R_{h}\right)}(u)(u,-1)=\frac{\partial^{2} R_{(1, h)}}{R_{(1, h)}^{2}-R_{h}}(u)(u,-1) \in \mathbb{R} \overrightarrow{u_{L}}
$$

Proof of Theorem 6. The steps of the proof are the same as those in the proof of Theorem 1. There is just some slight changes in formulas. For the convenience of the reader, we resume below the different steps of the proof.

In this proof, $\nabla_{H}$ (resp. $\nabla_{L}$ ) stands for the gradient on $\mathbf{H}^{2}$ (resp. $\mathbb{L}^{3}$ ), $\Delta_{H}$ for the Laplace-Beltrami operator on $\mathbf{H}^{2}$, and $\square$ for the d'Alembertian (or wave operator) on $\mathbb{L}^{3}$. Let $\left(x_{1}, x_{2}, x_{3}\right)$ be the standard coordinates on $\mathbb{L}^{3}$. For all differentiable function $\psi: \mathbb{L}^{3} \rightarrow \mathbb{R}$ and all $x \in \mathbb{L}^{3}, \nabla_{L} \psi(x)$ thus denote the vector with entries $\left(\frac{\partial \psi}{\partial x_{1}}(x), \frac{\partial \psi}{\partial x^{2}}(x),-\frac{\partial \psi}{\partial x_{3}}(x)\right)$ in $\mathbb{L}^{3}$. For its part, the d'Alembertian has the form $\square:=\frac{\partial^{2}}{\partial x_{1}^{2}}+\frac{\partial^{2}}{\partial x_{2}^{2}}-\frac{\partial^{2}}{\partial x_{3}^{2}}$. Besides, the Laplace-Beltrami operator of a vector function $X: \mathbf{H}^{2} \rightarrow \mathbf{M}^{4}, u \mapsto\left(X_{i}(u)\right)_{i=1}^{4}$ is understood to be the vector function $\Delta_{H} X: \mathbf{H}^{2} \rightarrow \mathbf{M}^{4}, u \mapsto\left(\Delta_{H} X_{i}(u)\right)_{i=1}^{4}$, where $\Delta_{H} X_{i}$ is the Laplace-Beltrami operator of the coordinate function $X_{i}$ in the intrinsic metric on $\mathbf{H}^{2},(1 \leq i \leq 4)$.

(i) For all $t \in \mathbb{R}$, let $x: \mathbf{H}^{2} \rightarrow \mathbf{M}^{4}$ be any $C^{\infty}$ map such that

$$
x(v) \in L_{h+t}(v):=\left\{l_{h+t}(v)\right\}+\mathbb{R} v_{L},
$$


for all $v \in \mathbf{H}^{2}$. Then there exists some $\lambda \in C^{\infty}\left(\mathbb{S}^{2} ; \mathbb{R}\right)$ such that $x$ is of the form

$$
\begin{aligned}
X_{\lambda}: & \mathbf{H}^{2} \rightarrow \mathbf{M}^{4} \\
& v \mapsto\left(\nabla_{H} h(v),-h(v)-t\right)+\lambda(v)(v,-1)=\left(x_{h}(v)+f(v) v,-f(v)-t\right),
\end{aligned}
$$

where $f:=h+\lambda$. For all $v \in \mathbf{H}^{2}$, we have:

$$
\Delta_{H} X_{\lambda}(v)=\left(\Delta_{H} x_{h}(v)+\Delta_{H}(f(v) v),-\left(\Delta_{H} f\right)(v)\right) .
$$

Now, $\Delta_{H} x_{h}=\Delta_{H}\left(\nabla_{L} \varphi\right)=\square\left(\nabla_{L} \varphi\right)=\nabla(\square \varphi)$, where $\varphi$ is the positively 1-homogeneous extension of $h$ to $\mathcal{U}=\left\{x \in \mathbb{L}^{3} \mid\langle x, x\rangle_{L}<0\right\}$, that is,

$$
\varphi(v):=\|x\|_{L} h\left(\frac{x}{\|x\|_{L}}\right),
$$

for $x \in \mathcal{U}$, where $\|x\|_{L}:=\sqrt{-\langle x, x\rangle_{L}}$. Indeed, $\nabla \varphi$ is positively 0-homogeneous on $\mathcal{U}$ and equal to $x_{h}$ on $\mathbf{H}^{2}$. Thus

$$
\left\langle\Delta_{H} x_{h}(v), v\right\rangle_{L}=-(\square \varphi)(v)=\left(-\Delta_{H} h+2 h\right)(v),
$$

for $v \in \mathbf{H}^{2}$, since $\square \varphi$ is positively -1-homogeneous on $\mathcal{U}$. Besides, we have:

$$
\begin{aligned}
\Delta_{H}(f(v) v) & =\left(\Delta_{H} f\right)(u) u+2\left(\left\langle\left(\nabla_{H} f\right)(v),\left(\nabla_{H} x_{i}\right)(v)\right\rangle_{L}\right)_{i=1}^{3}+f(v)\left(\Delta_{H} i d_{\mathbf{H}^{2}}\right)(v) \\
& =\left(\Delta_{H} f+2 f\right)(v) v+2\left(\nabla_{H} f\right)(v),
\end{aligned}
$$

for all $v \in \mathbf{H}^{2}$. Therefore, we have:

$$
\begin{aligned}
\left\langle\Delta_{H} X_{\lambda}(v), v_{M}\right\rangle_{M}=0 & \Leftrightarrow\left(-\Delta_{H} h+2 h\right)(v)-\left(\Delta_{H} f+2 f\right)(u)+\left(\Delta_{H} f\right)(v)=0 \\
& \Leftrightarrow-\left(-\Delta_{H} h+2 h\right)(u)-2(h+\lambda)(v)=0 \\
& \Leftrightarrow \lambda=-\frac{1}{2}\left(\Delta_{H} h\right)(v)
\end{aligned}
$$

for all $v \in \mathbf{H}^{2}$. Thus

$$
x(v)=X_{\lambda}(v)=l_{h+t}(v)-\frac{\left(\Delta_{H} h\right)(v)}{2}(v,-1)=l_{h+t}(v)+\delta(\partial h)(v),
$$

for all $v \in \mathbf{H}^{2}$.

(ii) We know that $x(v)=\left(x_{h}(v), t\right)-R_{(-1, h)}(v)(v,-1)$ for all $v \in \mathbf{H}^{2}$, where $R_{(-1, h)}:=-h+\frac{\Delta_{H} h}{2}=\frac{1}{2}\left(R_{1}+R_{2}\right)$ is the mean radius of principal curvature of $\mathcal{H}_{h}$. From this we deduce that

$$
\left(T_{v} x\right)(w)=\left(\left(T_{v} x_{h}\right)(w), 0\right)-\left(d R_{(-1, h)}\right)_{v}(w)(v,-1)-R_{(-1, h)}(v)(w, 0) ;
$$

and thus that

$$
\left\langle\left(T_{v} x\right)(w),\left(T_{v} x\right)(w)\right\rangle_{M}=\left\langle\left(T_{u} x_{h}\right)(v)-R_{(1, h)}(u) v,\left(T_{u} x_{h}\right)(v)-R_{(1, h)}(u) v\right\rangle,
$$


for all $w \in T_{v} \mathbf{H}^{2}$. Now, considering an orthonormal basis $\left(e_{1}, e_{2}\right)$ of $T_{v} \mathbf{H}^{2}$ made of eigenvectors of $T_{v} x_{h}$ (i.e., $\left(T_{v} x_{h}\right)\left(e_{i}\right)=R_{i}(v) e_{i}$ for $\left.i \in\{1,2\}\right)$, we conclude that: $\forall w \in T_{v} \mathbf{H}^{2}$,

$$
\left(x^{*} g\right)_{v}(w, w):=\left\langle\left(T_{v} x\right)(w),\left(T_{v} x\right)(w)\right\rangle_{L}=\frac{1}{4}\left(R_{1}-R_{2}\right)(u)^{2} g_{H}(v, v) .
$$

(iii) We know that: $\forall v \in \mathbf{H}^{2}, x(v)=\left(x_{h}(v),-t\right)-f(v)(v,-1)$, where $f=R_{(-1, h)}$. From this, we deduce that: $\forall v \in \mathbf{H}^{2}$,

$$
\left(\Delta_{H} x\right)(v)=\left(\Delta_{H} x_{h}(v)-\Delta_{H}(f(v) v),\left(\Delta_{H} f\right)(v)\right) .
$$

Now: $\forall v \in \mathbf{H}^{2}, \Delta_{H}(f(v) v)=\left(\Delta_{H} f+2 f\right)(v) v+2\left(\nabla_{H} f\right)(v)$, (see above the proof of $(i))$. Besides, $\Delta_{H} x_{h}=\Delta_{H}\left(\nabla_{L} \varphi\right)=\square\left(\nabla_{L} \varphi\right)=\nabla(\square \varphi)$, where $\varphi$ is the positively 1-homogeneous extension of $h$ to $\mathcal{U}=\left\{x \in \mathbb{L}^{3} \mid\langle x, x\rangle_{L}<0\right\}$ (see ib.), so that $\Delta_{H} x_{h}(v)=\nabla_{H}(\square \varphi)(v)+(\square \varphi)(v) v=2\left(\nabla_{H} f(v)+f(v) v\right)$ for all $v \in \mathbf{H}^{2}$, since $\square \varphi$ is positively -1 -homogeneous on $\mathcal{U}$, and equal to $2 f$ on $\mathbf{H}^{2}$. Therefore: $\forall v \in \mathbf{H}^{2}$,

$$
\left(\Delta_{H} x\right)(v)=-\left(\Delta_{H} f\right)(v)(v,-1)=-\left(\Delta_{H} R_{(-1, h)}\right)(v)(v,-1) \in \mathbb{R} \overrightarrow{v_{M}} .
$$

Above, we have demonstrated that $x^{*} g=\frac{1}{4}\left(R_{1}-R_{2}\right)(v)^{2} g_{H}=\left(R_{(-1, h)}^{2}-R_{h}\right) g_{H}$, where $R_{h}:=R_{1} R_{2}$ is the curvature function of $\mathcal{H}_{h}$, and that $\left(\Delta_{H} x\right)(v)=$ - $\left(\Delta_{H} R_{(-1, h)}\right)(v)(v,-1)$ for all $v \in \mathbf{H}^{2}$. Therefore

$$
\left(\Delta_{x^{*} g} x\right)(v)=-\frac{\Delta_{H} R_{(-1, h)}}{R_{(-1, h)}^{2}-R_{h}}(v)(v,-1)
$$

outside umbilical points of $\mathcal{H}_{h}$ (i.e., for all $v \in \mathbf{H}^{2}$ such that $R_{1}(v) \neq R_{2}(v)$ ). We also know that the mean vector field of any surface $X: \Omega \subset \mathbf{H}^{2} \rightarrow \mathbf{M}^{4}$, $v \mapsto\left(X_{i}(v)\right)_{i=1}^{4}$ is given by $\vec{H}_{X}=\frac{1}{2}\left(\Delta_{H} X\right)=\frac{1}{2}\left(\Delta_{H} X_{i}\right)_{i=1}^{4}$, where $\Delta_{H} X_{i}$ is the Laplace-Beltrami operator of the coordinate function in the intrinsic metric of the surface. Therefore: $\forall v \in \mathbf{H}^{2} \backslash\left\{v \in \mathbf{H}^{2} \mid R_{1}(v)=R_{2}(v)\right\}$,

$$
\vec{H}_{x}(v)=-\frac{\Delta_{H} R_{(-1, h)}}{2\left(R_{(-1, h)}^{2}-R_{h}\right)}(v)(v,-1)=\frac{\partial^{2} R_{(-1, h)}}{R_{(-1, h)}^{2}-R_{h}}(v)(v,-1) \in \mathbb{R} \overrightarrow{v_{M}}
$$

Let us now turn to the proofs of Theorem 2 and Theorem 5.

Proof of Theorem 2. Since the mixed curvature function $R: C^{\infty}\left(\mathbb{S}^{2}, \mathbb{R}\right)^{2} \rightarrow \mathbb{R}$, $(f, g) \mapsto R_{(f, g)}$ is bilinear and symmetric, it follows that the same holds true for the mixed Laguerre area 


$$
\begin{aligned}
L: \quad(\partial \mathcal{H})^{2} & \mapsto \mathbb{R} \\
(\partial f, \partial g) & \mapsto \int_{\mathbb{S}^{2}}\left(R_{(1, f)} R_{(1, g)}-R_{(f, g)}\right) d \sigma .
\end{aligned}
$$

Furthermore, for all $\partial h \in \partial \mathcal{H}$, we have:

$$
(s(\partial h)=0) \Longleftrightarrow\left(\mathcal{H}_{h} \text { is totally umbilical }\right) \Longleftrightarrow\left(\partial h=0_{\partial \mathcal{H}}\right) .
$$

This completes the proof.

Proof of Theorem 5. For any $h \in C^{4}\left(\mathbb{S}^{2}, \mathbb{R}\right)$, we know from (ii) of Theorem 1 (which remains true under our present smoothness assumption) that $x^{*} g=$ $\frac{1}{4}\left(R_{1}-R_{2}\right)^{2} g_{S}$, where $g_{S}$ is the standard metric on $\mathbb{S}^{2}, g$ the first fundamental form on $x\left(\mathbb{S}^{2}\right), x^{*} g$ the pullback of $g$ along $x$, and $R_{1}(u), R_{2}(u)$ the principal radii of curvature of $\mathcal{H}_{h}$ at $x_{h}(u)$ for all $u \in \mathbb{S}^{2}$. Therefore

$$
s(\partial h)=-L(\partial h)=-\frac{1}{4} \int_{\mathbb{S}^{2}}\left(R_{1}-R_{2}\right)^{2} d \sigma .
$$

Now $R_{1}=\lambda_{1}+h$ and $R_{2}=\lambda_{2}+h$, where $\lambda_{1}(u), \lambda_{2}(u)$ denote the eigenvalues of the Hessian of $h$ at $u$ for all $u \in \mathbb{S}^{2}$. Hence:

$$
s(\partial h)=-\frac{1}{4} \int_{\mathbb{S}^{2}}\left(\lambda_{1}-\lambda_{2}\right)^{2} d \sigma=\frac{1}{4} \int_{\mathbb{S}^{2}}\left(4\left(\Delta_{22} h\right)-\left(\Delta_{S} h\right)^{2}\right) d \sigma,
$$

where $\Delta_{2}$ and $\Delta_{22}$ are respectively the Laplace-Beltrami operator and the Monge-Ampère operator on $\mathbb{S}^{2}$ (that is, respectively the sum and the product of the eigenvalues $\lambda_{1}, \lambda_{2}$ of the of $h$ ). From the equality

$$
\int_{\mathbb{S}^{2}} R_{h} d \sigma=\int_{\mathbb{S}^{2}} h \cdot R_{(1, h)} d \sigma
$$

which is a direct consequence of the symmetry of the mixed volume, we obtain

$$
\int_{\mathbb{S}^{2}}\left(\Delta_{22} h\right) d \sigma=-\frac{1}{2} \int_{\mathbb{S}^{2}} h\left(\Delta_{S} h\right) d \sigma,
$$

after development and simplification, and then

$$
\int_{\mathbb{S}^{2}}\left(\Delta_{22} h\right) d \sigma=\frac{1}{2} \int_{\mathbb{S}^{2}}(\nabla h)^{2} d \sigma=\int_{\mathbb{S}^{2}}(\overrightarrow{\partial h})^{2} d \sigma
$$

by integrating by parts. It follows that:

$$
s(\partial h)=\int_{\mathbb{S}^{2}}\left((\overrightarrow{\partial h})^{2}-\left(\partial^{2} h\right)^{2}\right) d \sigma
$$

Note that the integration by parts formula for functions $f, g \in C^{2}\left(\mathbb{S}^{2} ; \mathbb{R}\right)$ can be written as 


$$
\int_{\mathbb{S}^{2}} \overrightarrow{\partial f} \cdot \overrightarrow{\partial g} d \sigma=\int_{\mathbb{S}^{2}} f\left(\partial^{2} g\right) d \sigma
$$

Therefore

$$
\begin{aligned}
\int_{\mathbb{S}^{2}}\left((\overrightarrow{\partial h})^{2}-\left(\partial^{2} h\right)^{2}\right) d \sigma & =\int_{\mathbb{S}^{2}}\left((\overrightarrow{\partial h})^{2}-\overrightarrow{\partial h} \cdot \overrightarrow{\partial\left(\partial^{2} h\right)}\right) d \sigma \\
& =\int_{\mathbb{S}^{2}} \overrightarrow{\partial h} \cdot \overrightarrow{\partial\left(h-\partial^{2} h\right)} d \sigma \\
& =\int_{\mathbb{S}^{2}} \overrightarrow{\partial h} \cdot \overrightarrow{\partial R_{(1, h)}} d \sigma
\end{aligned}
$$

since $R_{(1, h)}=h+\frac{\left(\Delta_{S} h\right)}{2}=h-\frac{\Delta h}{2}=h-\partial^{2} h$.

\section{Further remarks and problems}

\subsection{Curvature function of marginally trapped hedgehogs}

In analogy to the cases of ordinary hedgehogs and convex bodies of $\mathbb{R}^{3}$, we will say that a marginally trapped hedgehog $\mathcal{H}_{\partial h}$ of $\mathbb{L}^{4}$ has the curvature function $R \overrightarrow{\partial h}: \mathbb{S}^{2} \rightarrow \mathbb{R}$ if its signed surface area measure

$$
\begin{aligned}
s_{\partial h}: \quad \mathcal{B}\left(\mathbb{S}^{2}\right) & \rightarrow \mathbb{R} \\
\omega & \longmapsto-\int_{\omega}\left(R_{(1, h)}^{2}-R_{h}\right) d \sigma,
\end{aligned}
$$

where $\mathcal{B}\left(\mathbb{S}^{2}\right)$ is the Borel algebra on $\mathbb{S}^{2}$, has $R_{\partial h}$ as a density with respect to spherical Lebesgue measure $\sigma$. In other words, $\mathcal{H}_{\partial h}$ has the curvature function $R_{\overrightarrow{\partial h}}=-\left(R_{(1, h)}^{2}-R_{h}\right)=-\frac{1}{4}\left(R_{1}-R_{2}\right)^{2}$, where $R_{1}$ and $R_{2}$ are the principal radii of curvature of $\mathcal{H}_{h}$.

Proposition 3. Let $h \in C^{\infty}\left(\mathbb{S}^{2} ; \mathbb{R}\right)$. The curvature function of the marginally trapped hedgehog $\mathcal{H}_{\partial h}$ of $\mathbb{L}^{4}$ can be expressed in the form

$$
R_{\overrightarrow{\partial h}}=\overrightarrow{\partial h}^{2}+\overrightarrow{\partial h} \cdot(\nabla \circ \operatorname{div})(\overrightarrow{\partial h})+\frac{1}{2}\left((\operatorname{div} \overrightarrow{\partial h})^{2}-(\operatorname{div} \circ \nabla)\left(\overrightarrow{\partial h}^{2}\right)\right),
$$

where div stands for the divergence operator on $\mathbb{S}^{2}$.

This expression of $R_{\overrightarrow{\partial h}}$ has to be compared with the following one, which gives an expression for the curvature function of an ordinary hedgehog $\mathcal{H}_{h}$ of $\mathbb{R}^{3}$ :

$$
R_{h}=h^{2}+h(\operatorname{div} \circ \nabla)(h)+\frac{1}{2}\left(\left(\operatorname{tr} H_{h}\right)^{2}-\operatorname{tr}\left(H_{h}^{2}\right)\right),
$$


where $t r$ stands for the trace operator and $H_{h}$ is the Hessian of $h$. Of course, as for ordinary hedgehogs of $\mathbb{R}^{3}$, we can define a mixed curvature function for marginally trapped hedgehogs of $\mathbb{L}^{4}$ :

$$
R:(\partial \mathcal{H})^{2} \rightarrow C^{\infty}\left(\mathbb{S}^{2} ; \mathbb{R}\right),(\overrightarrow{\partial f}, \overrightarrow{\partial g}) \longmapsto R_{(\overrightarrow{\partial f}, \overrightarrow{\partial g})}:=\left(R_{(f, g)}-R_{(1, f)} R_{(1, g)}\right)
$$

Proof of Proposition 3. We know that

$$
R_{\overrightarrow{\partial h}}=-\frac{1}{4}\left(\lambda_{1}-\lambda_{2}\right)^{2}=\frac{1}{4}\left(\left(\operatorname{tr} H_{h}\right)^{2}-2 \operatorname{tr}\left(H_{h}^{2}\right)\right),
$$

where $\lambda_{1}$ and $\lambda_{2}$ are the eigenvalues of $H_{h}$. Now, in the present case, the classical Bochner-Lichnerowicz-Weitzenböck formula can be rewritten in the form

$$
\Delta_{S}\left(\overrightarrow{\partial h}^{2}\right)=2 \overrightarrow{\partial h} \cdot(\nabla \circ \operatorname{div})(\overrightarrow{\partial h})+\operatorname{tr}\left(H_{h}^{2}\right)+2 \overrightarrow{\partial h}^{2},
$$

where $\Delta_{S}=\operatorname{div} \circ \nabla$ is the Laplace-Beltrami operator on $\mathbb{S}^{2}$. Combining the above formulas yields the desired result.

Now let us mention briefly how the curvature function of marginally trapped hedgehogs of $\mathbb{L}^{4}$ is also involved in the volume of focal surfaces of ordinary hedgehogs of $\mathbb{R}^{3}$. Let $h \in C^{\infty}\left(\mathbb{S}^{2} ; \mathbb{R}\right)$. The focal surface, say $\mathcal{F}_{h}$, of $\mathcal{H}_{h}$ is defined as the locus of the centers of principal curvature of $\mathcal{H}_{h}$ (or, which is equivalent, as the envelope of its normal lines). In [8], we defined the volume of $\mathcal{F}_{h}$ by:

$$
v(\nabla h):=-\int_{\mathbb{R}^{3}} i_{\mathcal{F}_{h}}(x) d x,
$$

where $i_{\mathcal{F}_{h}}(x):=1-\frac{1}{2} \nu_{h}(x)$, denoting by $\nu_{h}(x)$ the number of oriented normal lines to $\mathcal{H}_{h}$ through $x$. Note that $\mathcal{F}_{h}$ and hence its volume only depend on $\nabla h$. In fact, from [8, Theorems 2 and 3], we have:

$$
v(\nabla h)=\frac{4}{3} \int_{\mathbb{S}^{2}}\left(-R_{\overrightarrow{\partial h}}\right)^{\frac{3}{2}} d \sigma
$$

and the geometric inequality

$$
4 L(\partial h)^{3} \leq 9 \pi v(\nabla h)^{2} .
$$

\subsection{Minkowski problem for marginally trapped hedgehogs}

When restricting to the class of convex bodies of $\mathbb{R}^{n+1}$ whose surface area measures have a density with respect to spherical Lebesgue measure, the classical Minkowski problem can be formulated as that of the existence, uniqueness and regularity of convex bodies of this class whose curvature function is prescribed 
(see e.g. [13, Section 8.2] for more details, results and a complete bibliography). This Minkowski problem admits a natural extension to hedgehogs of $\mathbb{R}^{n+1}$, which we considered in [8]. The extension to hedgehogs is much more difficult since it involves the study of a Monge-Ampère equation of mixed type (instead of a Monge-Ampère equation of elliptic type in the convex case). Since we have defined the curvature function $R_{\overrightarrow{\partial h}}$ of any marginally trapped hedgehog $\mathcal{H}_{\partial h}$ of $\mathbb{L}^{4}$ by analogy to the cases of hedgehogs and convex bodies of $\mathbb{R}^{3}$, it is now natural to consider the analogue of the Minkowski problem for marginally trapped hedgehogs (modulo some slight changes in the statement, as in the Minkowski problem for ordinary hedgehogs, due for instance to the fact that for any $h \in C^{\infty}\left(\mathbb{S}^{2} ; \mathbb{R}\right), \partial(-h)$ and $\partial h$ are the respective support differentials of two marginally trapped hedgehogs $\mathcal{H}_{\partial(-h)}$ and $\mathcal{H}_{\partial h}$ of $\mathbb{L}^{4}$ that have the same curvature function $R_{\overrightarrow{\partial(-h)}}=R_{\overrightarrow{\partial h}}$ and are such that

$$
\mathcal{H}_{\partial(-h)}=s\left(\mathcal{H}_{\partial h}\right)
$$

where $s$ is the symmetry with respect to the origin in $\mathbb{L}^{4}$ ).

This problem is certainly very difficult since to solve it properly, it would be necessary in particular to know if any convex closed and sufficiently smooth surface of $\mathbb{R}^{3}$ admits at least two umbilical points, that is, to have a complete solution to the Caratheodory conjecture.

\section{References}

[1] W. Blaschke, Vorlesungen über Differentialgeometrie und geometrische Grundlagen von Einsteins Relativitätstheorie, Vol. 3. Bearbeitet von G. Thomsen, J. Springer, Berlin, 1929.

[2] T. E. Cecil, Lie sphere geometry. With application to submanifolds. 2nd ed., Springer-Verlag, New York, 2008.

[3] B. Y. Chen, Pseudo-Riemannian geometry, $\delta$-invariants and applications, Word Scientific Publishing Co. Pte. Ltd., Hackensack, NJ, 2011.

[4] F. Fillastre, Fuchsian convex bodies: basics of Brunn-Minkowski theory. Geom. Funct. Anal. 23, (2013), 295-333.

[5] H. Geppert, Über den Brunn-Minkowskischen Satz, Math. Z. 42 (1937), 238-254.

[6] R. Langevin, G. Levitt, H. Rosenberg, Hérissons et multihérissons (enveloppes paramétrées par leur application de Gauss). In: Singularities, Banach Center Publ. 20, Warsaw, PWN, 1988, 245-253.

[7] Y. Martinez-Maure, De nouvelles inégalités géométriques pour les hérissons. Arch. Math. 72 (1999), 444-453. 
[8] Y. Martinez-Maure, Dérivation des surfaces convexes de $\mathbb{R}^{3}$ dans l'espace de Lorentz et étude de leurs focales, C. R. Acad. Sci. Paris, Sér. I, 348 (2010), 1307-1310.

[9] Y. Martinez-Maure, Uniqueness results for the Minkowski problem extended to hedgehogs. Cent. Eur. J. Math. 10, 2012, 440-450.

[10] Y. Martinez-Maure, Hedgehog theory via Euler Calculus, Beitr. Algebra Geom. 56 (2015), 397-421.

[11] Y. Martinez-Maure, Plane Lorentzian and Fuchsian hedgehogs, Canad. Mathematical Bulletin 58 (2015), 561-574.

[12] R. Penrose, Gravitational collapse and space-time singularities, Phys. Rev. Lett., 14 (1965), 57-59.

[13] R. Schneider, Convex Bodies: The Brunn-Minkowski Theory, 2nd expanded ed., Encyclopedia of Mathematics and its Applications, Cambridge University Press, Cambridge, 2014.

[14] C. Stadtmüller, Metric contact manifolds and their Dirac operators. Diplomarbeit. Humboldt Universität zu Berlin, Institut fûr Mathematik, 2011.

Y. Martinez-Maure

Institut Mathématique de Jussieu - Paris Rive Gauche

UMR 7586 du CNRS

Bâtiment Sophie Germain

Case 7012

75205 Paris Cedex 13

France 